\title{
Article \\ Thermochemistry, Bond Energies and Internal Rotor Potentials of Acetic Acid Hydrazide, Acetamide, N-Methyl Acetamide (NMA) and Radicals
}

\author{
Sumit Charaya and Joseph W. Bozzelli *(D)
}

check for updates

Citation: Charaya, S.; Bozzelli, J.W.

Thermochemistry, Bond Energies and Internal Rotor Potentials of Acetic Acid Hydrazide, Acetamide, $N$-Methyl Acetamide (NMA) and Radicals. Thermo 2021, 1, 15-31. https://doi.org/10.3390/thermo1010002

Academic Editor: Johan Jacquemin

Received: 24 November 2020

Accepted: 19 February 2021

Published: 2 March 2021

Publisher's Note: MDPI stays neutral with regard to jurisdictional claims in published maps and institutional affiliations.

Copyright: (c) 2021 by the authors. Licensee MDPI, Basel, Switzerland. This article is an open access article distributed under the terms and conditions of the Creative Commons Attribution (CC BY) license (https:/ / creativecommons.org/licenses/by/ $4.0 /)$.
Department of Chemistry and Environmental Science, New Jersey Institute of Technology, Newark, NJ 07102, USA; sc377@njit.edu

* Correspondence: bozzelli@njit.edu; Tel.: +1-973-596-3459; Fax: +1-973-596-3586

Abstract: Structures, thermochemical properties, bond energies, and internal rotation potentials of acetic acid hydrazide $\left(\mathrm{CH}_{3} \mathrm{CONHNH}_{2}\right)$, acetamide $\left(\mathrm{CH}_{3} \mathrm{CONH}_{2}\right)$, and $\mathrm{N}$-methyl acetamide $\left(\mathrm{CH}_{3} \mathrm{CONHCH}_{3}\right)$, and their radicals corresponding to the loss of hydrogen atom, have been studied. Gas-phase standard enthalpies of formation and bond energies were calculated using the DFT methods B3LYP / 6-31G(d,p), B3LYP / 6-31G(2d,2p) and the composite CBS-QB3 methods employing a series of work reactions further to improve the accuracy of the $\Delta \mathrm{H}_{\mathrm{f}}{ }^{\circ}(298 \mathrm{~K})$. Molecular structures, vibration frequencies, and internal rotor potentials were calculated at the DFT level. The parent molecules standard formation enthalpies of $\mathrm{CH}_{3}-\mathrm{C}=\mathrm{ONHNH}_{2}, \mathrm{CH}_{3}-\mathrm{C}=\mathrm{ONH}_{2}$, and $\mathrm{CH}_{3}-\mathrm{C}=\mathrm{ONHCH}_{3}$ were evaluated as $-27.08,-57.40$, and $-56.48 \mathrm{kcal} \mathrm{mol}^{-1}$, respectively, from the CBS-QB3 calculations. Structures, internal rotor potentials, and $\mathrm{C}-\mathrm{H}$ and $\mathrm{N}-\mathrm{H}$ bond dissociation energies are reported. The DFT and the CBS-QB3 enthalpy values show close agreement, and this accord is attributed to the use of isodesmic work reactions for the analysis. The agreement also suggests this combination of the B3LYP/work reaction approach is acceptable for larger molecules. Internal rotor potentials for the amides are high, ranging from 16 to $22 \mathrm{kcal} \mathrm{mol}^{-1}$.

Keywords: thermochemistry; enthalpy of formation; bond energy; acetohydrazide; acetamide and N-Methyl acetamide

\section{Introduction}

Thermochemical and spectroscopic investigation of N-methyl Acetamide (NMA) and other substituted amides are considered as model compounds for the peptide bonds in proteins; understanding their thermochemistry can provide information about the secondary structure of proteins in the gas phase as well as inferences in solution and helpful information toward understanding kinetics. There are no studies that we are aware of that have targeted these molecules' thermochemical properties and bond energies.

There are several reasons for interest in the structure and chemistry of these amide systems. These include: (i) clear understanding of the NMA structure is considered as the basis for understanding the geometric constraints imposed by the peptide linkages that determine, at least partly, the protein structure; (ii) detailed understanding of NMA spectroscopic features is assumed as the fundamental basis for spectroscopic methods to monitor protein structure and dynamics [1]. Both of these properties are of interest for future applications, only if both structures and spectroscopic properties of NMA are observed in the natural environment of the biological system(s).

There are many infrared (I.R.), and Raman experiments that have focused on the spectral region spanned by the three amide bands of NMA, particularly in the easily detectable I.R. amide I regime that overlaps with the C.O. stretch. In water (aq), the C.O. stretch responds to water molecules' presence by forming hydrogen bonds, and the resulting frequency shift can be used to assess the dynamics of protein-solvent interactions [2-9]. 
Similarly, the amide II and amide III bands, which overlap with the N.H. in-plane wagging motion, can be used to describe the interaction between C.O. and H.N., which are part of a protein backbone. The amide hydrogen can also form a hydrogen bond with the solvent $\left(\mathrm{H}_{2} \mathrm{O} \cdot \mathrm{HN}\right)$, and the corresponding frequency shift provides further information on protein behavior in an aqueous solution [10].

The structural stability of acetohydrazide $\mathrm{CH}_{3}-\mathrm{CO}-\mathrm{NH}-\mathrm{NH}_{2}$ was investigated by DFT-B3LYP and ab initio MP2 calculations with a $6-311+\mathrm{G}^{* *}$ basis set. The $\mathrm{C}-\mathrm{N}$ rotational barrier in the molecule was calculated to be $26 \mathrm{kcal} \mathrm{mol}^{-1}$, which suggested the planar sp2 nature of the nitrogen atom of the central N.H. moiety with the two-fold barrier. The $\mathrm{N}$ atom of the terminal $\mathrm{NH}_{2}$ group was predicted to prefer the pyramidal sp3 structure with an inversion barrier of 7-8 $\mathrm{kcal} \mathrm{mol}^{-1}$. The molecule was predicted to have a trans-syn ( $\mathrm{N}-\mathrm{H}$ bond is trans with respect to $\mathrm{C}=\mathrm{O}$ bond and the $\mathrm{NH}_{2}$ moiety is syn to $\mathrm{C}-\mathrm{N}$ bond) conformation as the lowest energy structure. The vibrational frequencies were computed at the B3LYP/6-311+G* level of theory and normal coordinate calculations were carried out for the trans-syn acetohydrazide. Complete vibrational assignments were made based on normal coordinate analyses and experimental infrared and Raman data [11].

The study of amide $\mathrm{C}-\mathrm{N}$ rotation barriers is important because amide $\mathrm{C}-\mathrm{N}$ bonds make up protein backbones. The preferred amide conformations play an important role in enzyme structure and the barrier to the rotation affects the rigidity of the structure. The rigidity of an enzyme's structure can affect its selectivity in binding substrates. Ab initio calculations by Jasien et al. [12] have been used to determine the gas-phase rotational barrier about the $\mathrm{C}-\mathrm{N}$ bond in acetamide. Their results indicate that the inclusion of polarization functions in the basis sets leads to a substantial decrease (ca. $5 \mathrm{kcal} \mathrm{mol}^{-1}$ ) in the calculated barrier height at the H.F.-SCF level. Electron correlation effects decrease the barrier by less than $1 \mathrm{kcal} \mathrm{mol}^{-1}$, while the addition of zero-point energy corrections changed the barrier height only slightly. Based upon the current $(\mathbf{D Z}+\mathbf{d} / \mathbf{S C F})$ calculations, the $0 \mathrm{~K}$ rotational barrier for acetamide is predicted to be $12.5 \mathrm{kcal} \mathrm{mol}^{-1}$. An investigation of the photolysis of acetamide was performed using light in the $250 \AA$ regions of the spectrum, where the goal was to break down the molecule into $\mathrm{CH}_{3}$ and $\mathrm{CONH}_{2}$ radicals. The authors reported this was probably accompanied by a second process yielding $\mathrm{CH}_{3} \mathrm{CN}$ and $\mathrm{H}_{2} \mathrm{O}$. Methyl radicals were observed to react with the parent acetamide and with the $\mathrm{CONH}_{2}$ radical to give methane as a product and to recombine yielding ethane. The $\mathrm{CONH}_{2}$ radicals were reported to decompose both spontaneously and thermally to give C.O. and $\mathrm{NH}_{2}$ radicals. The subsequent reaction of the $\mathrm{NH}_{2}$ radicals with Acetamide gives ammonia. In a separate experiment with acetone as a photo methyl radical source, the activation energy for the abstraction of hydrogen by methyl radical was found to be $9.2 \mathrm{kcal} \mathrm{mol}^{-1}$ [13].

The importance of reliable and accessible thermochemical data (enthalpies of formation, entropies, and heat capacities) is universally accepted among scientists and engineers. This work provides thermochemical data for acetic acid hydrazide, acetamide, and Nmethyl acetamide and their radicals corresponding to the loss of hydrogen atoms through the use of computational chemistry.

\section{Computational Methods}

Density functional theory and composite calculations via series of isodesmic reactions: the structure and thermochemical parameters of $\mathrm{CH}_{3} \mathrm{CONHNH}_{2}, \mathrm{CH}_{3} \mathrm{CONH}_{2}$, and $\mathrm{CH}_{3} \mathrm{CONHCH}_{3}$ are based on the density functional and composite ab initio levels using Gaussian 98 [14] and Gaussian 09 [15]. Computation levels include B3LYP/6-31G(d,p), B3LYP/6-31G(2d,2p). These methods combine the three-parameter Becke exchange functional B3 [16], with the Lee-Yang-Parr correlation functional, LYP, [17], and are used here with the 6-31G $(d, p)$ basis set. B3LYP/6-31G $(d, p)$ is chosen because it is computational, economical, and, thus, possibly applicable to larger molecules [18]. Energies are further refined using the procedures of the complete basis method developed by Petersson and co-workers, CBS-QB3 [19]. The CBS-QB3 method is utilized for improved energies and serves to check the DFT calculations. CBS models, a series of calculations made on a 
defined geometry, and a complete basis set model chemistry including corrections for basis set truncation errors. These methods show accuracy in structure and energy that requires convergence in basis set size and the degree of correlation [18].

Standard enthalpies of formation for stable species are calculated using the total energies at B3LYP / 6-31G $(d, p), B 3 L Y P / 6-31 G(2 d, 2 p)$, and CBS-QB3 levels with work reactions that are isodesmic in most cases. Isodesmic reactions conserve the number and type of bonds on both sides of an equation. The use of a work reaction with similar bonding on both sides of an equation results in a cancellation of calculation error and improves the accuracy for energy analysis [20]. The reported enthalpy values can be compared with the known enthalpies of several molecules in the system to serve as a calibration on the thermochemistry.

Contributions to $\mathrm{S}^{\circ} 298$ and $\mathrm{CP}^{\circ}(\mathrm{T})$ of each species are calculated using the "SMCPS" (Statistical Mechanics for Heat Capacity and Entropy Cp and S) program, which incorporates the frequencies, moments of inertia, mass, symmetry, number of optical isomers, from the Gaussian calculation. It also incorporates frequency corrections. Contributions from hindered internal rotors to $S^{\circ} 298$ and $C p(T)$ are determined using the "VIBIR" program. The hindered rotor corrections to the $S^{\circ} 298$ and $C p(T)$ are obtained by adding the $S$ and $\mathrm{C} p$ values, respectively, obtained by employing the VIBIR program to those obtained from SMCPS.

\section{Results and Discussion}

Optimized, lowest energy structures of the parent molecules-acetohydrazide, acetamide, and $N$-methyl acetamide are shown in Figure 1.

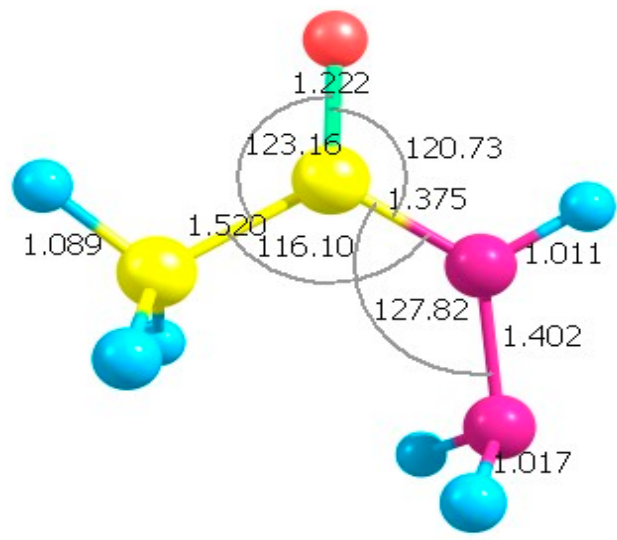

$\mathrm{CH}_{3}-\mathrm{C}=\mathrm{ONHNH}_{2}$

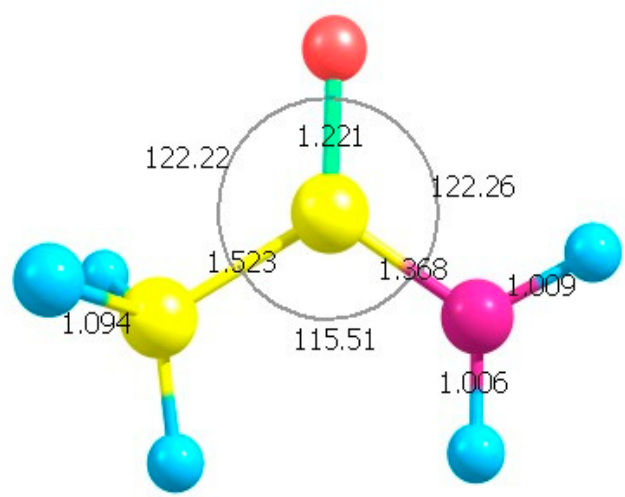

$\mathrm{CH}_{3}-\mathrm{C}=\mathrm{ONH}_{2}$

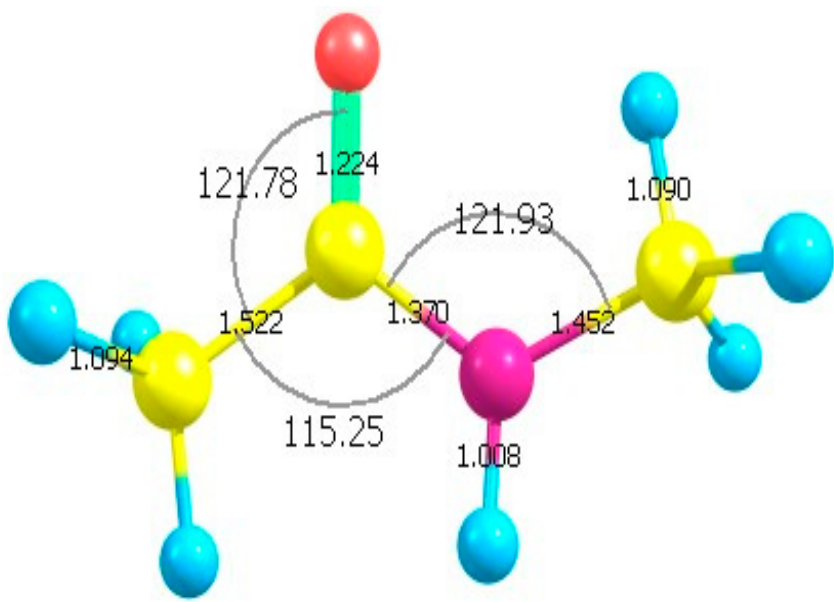

$\mathrm{CH}_{3}-\mathrm{C}=\mathrm{ONHCH}_{3}$

Figure 1. Optimized structures of the parent molecules $\left(\mathrm{CH}_{3}-\mathrm{C}=\mathrm{ONHNH}_{2}, \mathrm{CH}_{3}-\mathrm{C}=\mathrm{ONH}_{2}\right.$, and $\left.\mathrm{CH}_{3}-\mathrm{C}=\mathrm{ONHCH}_{3}\right)$ at B3LYP/6-31G $(\mathrm{d}, \mathrm{p})$ level. All show the nitrogen bonded to the carbonyl in a sp2 configuration. 
The torsional potentials of $\mathrm{CH}_{3}-\mathrm{C}=\mathrm{ONHNH}_{2}$ and $\mathrm{CH}_{3}-\mathrm{C}=\mathrm{ONHCH}_{3}$ (vide infra) show that corresponding anti- and syn-isomers respective to $\mathrm{H}_{8} \mathrm{~N}_{7}-\mathrm{C}_{5} \mathrm{O}_{6}$ dihedral angles (see Figure 3). The anti-acetohydrazide has a near $-4.86 \mathrm{kcal} \mathrm{mol}^{-1}$ lower energy than for the syn configuration, with a $20.7 \mathrm{kcal} \mathrm{mol}^{-1}$ barrier to the internal rotation converting the two-isomer configuration.

In contrast, the syn- $N$-methyl acetamide has a near $-2.50 \mathrm{kcal} \mathrm{mol}^{-1}$ lower energy than for the anti-configuration, with a $19.3 \mathrm{kcal} \mathrm{mol}^{-1}$ barrier. The internal rotation energies for these syn-anti isomerizations are high, typically greater than $13 \mathrm{kcal} \mathrm{mol}^{-1}$ (see below). This isomerization does not occur at standard temperature, and the isomers should be considered as different molecules in their thermochemistry and probably in their reactions.

The optimized geometries at the B3LYP/6-31G $(\mathrm{d}, \mathrm{p})$ density functional calculation level for $\mathrm{CH}_{3}-\mathrm{C}=\mathrm{ONHNH}_{2}, \mathrm{CH}_{3}-\mathrm{C}=\mathrm{ONH}_{2}$, and $\mathrm{CH}_{3}-\mathrm{C}=\mathrm{ONHCH}_{3}$ are presented in the Supplementary Materials [SM]. The Geometric Parameters (See Section SM0, Tables S1-S3 of the SM) are listed.

\subsection{Enthalpies of Formation of the Parent Molecules}

Enthalpies of formation $\left(\Delta \mathrm{H}_{\mathrm{f}}{ }^{\circ} 298\right)$ of the parent molecules have been determined using corresponding $\Delta$ Hrxn (298) from the enthalpy of reaction in the isodesmic work reactions and calculated enthalpies of each species. The standard enthalpies of formation of the reference molecules at $298.15 \mathrm{~K}$ and the calculated $\Delta \mathrm{Hrxn}^{\circ} 298$ are used to calculate the $\Delta_{\mathrm{f}} \operatorname{Hrxn}^{\circ} 298$ of the target molecule; the enthalpies are summarized in Table 1.

$$
\Delta \operatorname{Hrxn}^{\circ} 298=\Sigma \mathrm{H}_{\mathrm{f}} \text { products }-\Sigma \mathrm{H}_{\mathrm{f}} \text { reactants }
$$

Table 1. Standard enthalpies of formation of reference species at $298.15 \mathrm{~K}$.

\begin{tabular}{|c|c|c|}
\hline Species & $\Delta \mathrm{Hf}^{\circ} 298\left(\mathrm{kcal} \mathrm{mol}^{-1}\right)$ & Reference No. \\
\hline $\mathrm{CH}_{3} \mathrm{NHNH}_{2}$ & 22.6 & \multirow{14}{*}{ NIST [21] } \\
\hline $\mathrm{CH}_{3} \mathrm{CONH}_{2}$ & $-56.96 \pm 0.19$ & \\
\hline $\mathrm{CH}_{3} \mathrm{CH}_{3}$ & $-20.04 \pm 0.1$ & \\
\hline $\mathrm{CH}_{3} \mathrm{CH}_{2} \mathrm{CH}_{3}$ & $-25.02 \pm 0.12$ & \\
\hline $\mathrm{NH}_{2} \mathrm{CONH}_{2}$ & $-56.29 \pm 0.29$ & \\
\hline $\mathrm{CH}_{3} \mathrm{COCH}_{3}$ & $-52.23 \pm 0.14$ & \\
\hline $\mathrm{NH}_{2} \mathrm{CH}_{2} \mathrm{CO}_{2} \mathrm{H}$ & $-93.3 \pm 1.1$ & \\
\hline $\mathrm{CH}_{3} \mathrm{CH}_{2} \mathrm{OH}$ & $-56 \pm 0.5$ & \\
\hline $\mathrm{CH}_{3} \mathrm{CO}_{2} \mathrm{H}$ & $-103.5 \pm 0.6$ & \\
\hline $\mathrm{CH}_{3} \mathrm{C} \cdot \mathrm{HCH}_{3}$ & $22.5 \pm 0.5$ & \\
\hline $\mathrm{NH} 3$ & $-10.98 \pm 0.084$ & \\
\hline $\mathrm{NH}_{2} \mathrm{~N} \cdot \mathrm{H}$ & 52.74 & \\
\hline $\mathrm{C} \cdot \mathrm{H}_{2} \mathrm{NH}_{2}$ & 36.69 & \\
\hline $\mathrm{CH}_{3} \mathrm{~N} \cdot \mathrm{H}$ & 44.84 & \\
\hline $\mathrm{NH}_{2} \mathrm{NH}_{2}$ & 23.18 & Dorofeeva [22] \\
\hline $\mathrm{CH}_{3} \mathrm{CH}_{2} \mathrm{NH}_{2}$ & $-11.35 \pm 0.14$ & Pedley [23] \\
\hline $\mathrm{N} \cdot \mathrm{H}_{2}$ & 44.5 & Anderson [24] \\
\hline $\mathrm{CH}_{3} \mathrm{OH}$ & $-47.97 \pm 3$ & Bozzelli [25] \\
\hline $\mathrm{C} \cdot \mathrm{H}_{2} \mathrm{CHO}$ & $4.4 \pm 0.84$ & \multirow{6}{*}{ ATcT [26] } \\
\hline $\mathrm{CH}_{3} \mathrm{C} \cdot \mathrm{HOH}$ & $-13.2 \pm 0.61$ & \\
\hline $\mathrm{C} \cdot \mathrm{H}_{2} \mathrm{OH}$ & $-3.9 \pm 0.33$ & \\
\hline $\mathrm{CH}_{3} \mathrm{CHO}$ & $-39.9 \pm 0.28$ & \\
\hline $\mathrm{CH}_{3} \mathrm{C} \cdot \mathrm{H}_{2}$ & $28.6 \pm 0.28$ & \\
\hline $\mathrm{CH}_{3} \mathrm{NH}_{2}$ & -4.6 & \\
\hline
\end{tabular}


The work reactions and the enthalpies obtained from three isodesmic reactions for the parent molecules are listed in Table 2. Comparing the values of the enthalpies of the parent molecules calculated by two DFT and the CBS-QB3 methods shows that the values obtained by the DFT methods method are in close agreement with those obtained by CBS-QB3 calculations. We recommend the values obtained by the CBS-QB3 because it is a composite method and is known to have higher accuracy. The agreement of the DFT values suggests that the use of B3LYP calculations with the 6-31G $(d, p)$ and 6-31G $(2 d, 2 p)$ basis sets coupled with work reactions results in the cancelation of error and provides reasonable results for these amide systems.

The recommended enthalpies of formation for the $\mathrm{CH}_{3}-\mathrm{C}=\mathrm{ONHNH}_{2}, \mathrm{CH}_{3}-\mathrm{C}=\mathrm{ONH}_{2}$, and $\mathrm{CH}_{3}-\mathrm{C}=\mathrm{ONHCH}_{3}$ molecules obtained in this study are: $-27.08 \mathrm{kcal} \mathrm{mol}^{-1},-57.40 \mathrm{kcal} \mathrm{mol}^{-1}$, and $-56.48 \mathrm{kcal} \mathrm{mol}^{-1}$ by the CBS-QB3 calculation method.

Moments of Inertia (See Section SM1, Table S4 of the SM), vibrational frequencies (Table S5 of the SM), and Mulliken Atomic Charges (Section SM2, Tables S6-S8 of the $\mathrm{SM}$ ) of $\mathrm{CH}_{3}-\mathrm{C}=\mathrm{ONHNH}_{2}, \mathrm{CH}_{3}-\mathrm{C}=\mathrm{ONH}_{2}$, and $\mathrm{CH}_{3}-\mathrm{C}=\mathrm{ONHCH}_{3}$ and their radicals are calculated and presented.

Table 2. Enthalpies of reaction at $298 \mathrm{~K}$ and calculated enthalpies of formation $\left(\Delta \mathrm{H}_{\mathrm{f}}{ }^{\circ} 298\right)$ of parent molecules.

\begin{tabular}{|c|c|c|c|}
\hline $\mathrm{CH}_{3} \mathrm{CONHNH}_{2}$ Units: $\left(\mathrm{kcal} \mathrm{mol}^{-1}\right)$ & B3LYP/631G(d,p) & B3LYP/6-31G(2d,2p) & CBS-QB3 \\
\hline $\mathrm{CH}_{3} \mathrm{CONHNH}_{2}+\mathrm{CH}_{3} \mathrm{NH}_{2} \rightarrow \mathrm{CH}_{3} \mathrm{NHNH}_{2}+\mathrm{CH}_{3} \mathrm{CONH}_{2}$ & -29.43 & -30.57 & -29.51 \\
\hline $\mathrm{CH}_{3} \mathrm{CONHNH}_{2}+\mathrm{CH}_{3} \mathrm{CH}_{2} \mathrm{OH} \rightarrow \mathrm{CH}_{3} \mathrm{CH}_{2} \mathrm{NH}_{2}+\mathrm{NH}_{2} \mathrm{CH}_{2} \mathrm{CO}_{2} \mathrm{H}$ & -26.45 & -26.10 & -25.29 \\
\hline $\mathrm{CH}_{3} \mathrm{CONHNH}+\mathrm{CH}_{3} \mathrm{CHO} \rightarrow \mathrm{NH}_{2} \mathrm{CONH}_{2}+\mathrm{CH}_{3} \mathrm{COCH}_{3}$ & -29.61 & -29.56 & -26.44 \\
\hline Standard Enthalpy—Average & -28.50 & -28.74 & -27.08 \\
\hline \multicolumn{4}{|l|}{$\mathrm{CH}_{3} \mathrm{CONH}_{2}$} \\
\hline $\mathrm{CH}_{3} \mathrm{CONH}_{2}+\mathrm{CH}_{3} \mathrm{CH}_{2} \mathrm{OH} \rightarrow \mathrm{CH}_{3} \mathrm{COOH}+\mathrm{CH}_{3} \mathrm{CH}_{2} \mathrm{NH}_{2}$ & -56 & -55.13 & -56.63 \\
\hline $\mathrm{CH}_{3} \mathrm{CONH}_{2}+\mathrm{CH}_{3} \mathrm{CH}_{3} \rightarrow \mathrm{CH}_{3} \mathrm{COCH}_{3}+\mathrm{CH}_{3} \mathrm{NH}_{2}$ & -58.46 & -57.08 & -55.90 \\
\hline $\mathrm{CH}_{3} \mathrm{CONH}_{2}+\mathrm{CH}_{3} \mathrm{NH}_{2} \rightarrow \mathrm{CH}_{3} \mathrm{NHNH}_{2}+\mathrm{CH}_{3} \mathrm{CHO}$ & -58.72 & -58.30 & -59.66 \\
\hline Standard Enthalpy-Average & -57.73 & -56.84 & -57.40 \\
\hline \multicolumn{4}{|l|}{$\mathrm{CH}_{3} \mathrm{CONHCH}_{3}$} \\
\hline $\mathrm{CH}_{3} \mathrm{CONHCH}_{3}+\mathrm{CH}_{3} \mathrm{NH}_{2} \rightarrow \mathrm{CH}_{3} \mathrm{CONH}_{2}+\mathrm{CH}_{3} \mathrm{CH}_{2} \mathrm{NH}_{2}$ & -55.31 & -56.04 & -55.05 \\
\hline $\mathrm{CH}_{3} \mathrm{CONHCH}_{3}+\mathrm{CH}_{3} \mathrm{NH}_{2} \rightarrow \mathrm{CH}_{3} \mathrm{COCH}_{3}+\mathrm{CH}_{3} \mathrm{NHNH}_{2}$ & -57.42 & -57.53 & -57.31 \\
\hline $\mathrm{CH}_{3} \mathrm{CONHCH}_{3}+\mathrm{NH}_{2} \mathrm{NH}_{2} \rightarrow \mathrm{CH}_{3} \mathrm{CONH}_{2}+\mathrm{CH}_{3} \mathrm{NHNH}_{2}$ & -55.85 & -57.22 & -57.08 \\
\hline Standard Enthalpy-Average & -56.19 & -56.93 & -56.48 \\
\hline
\end{tabular}

\subsection{Radicals Corresponding to the Loss of a Hydrogen Atom}

Optimized, lowest energy structures of the radicals derived from the target parent molecules are illustrated in Figure 2.

The radicals from all three parent molecules show anti-structures relative to the carbonyl group for the low energy conformation where the radical sites are on the carbons and the nitrogen, not adjacent to the carbonyl. When the radical site is on the nitrogen atom adjacent to the carbonyl, all three radicals show that the syn conformer is the lowest energy structure. 


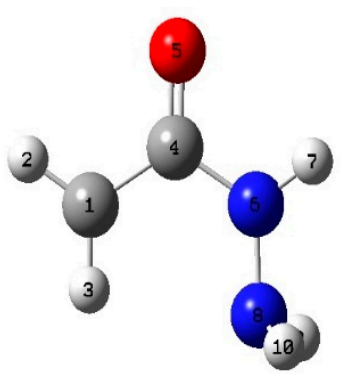

$\mathrm{C} \cdot \mathrm{H}_{2}-\mathrm{C}=\mathrm{ONHNH}_{2}$

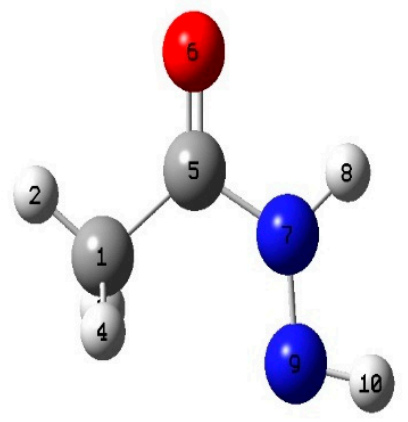

$\mathrm{CH}_{3}-\mathrm{C}=\mathrm{ONHN} \cdot \mathrm{H}$

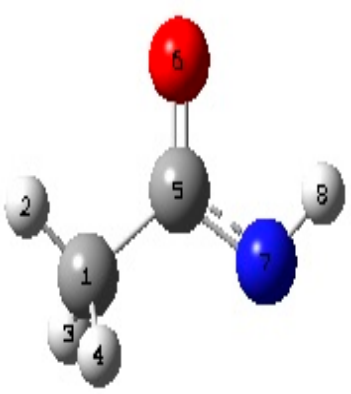

$\mathrm{CH}_{3}-\mathrm{C}=\mathrm{ON} \cdot \mathrm{H}$

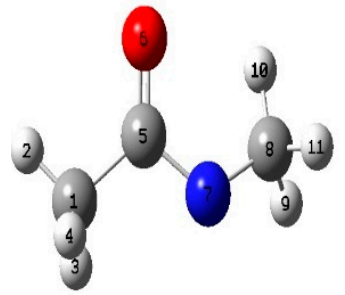

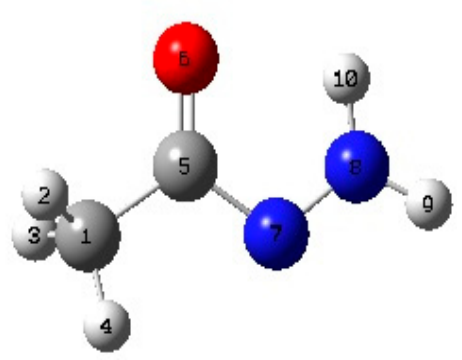

$\mathrm{CH}_{3}-\mathrm{C}=\mathrm{ON} \bullet \mathrm{NH}_{2}$

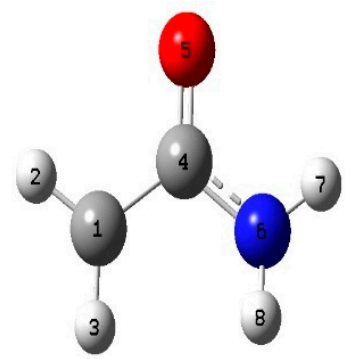

$\mathrm{C} \cdot \mathrm{H}_{2}-\mathrm{C}=\mathrm{ONH}_{2}$

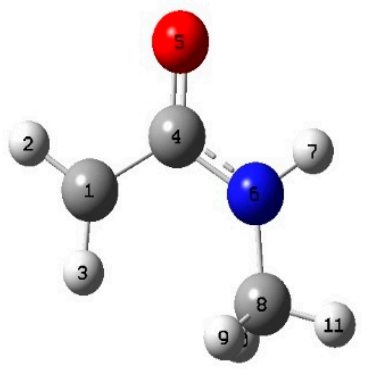

$\mathrm{C} \cdot \mathrm{H}_{2}-\mathrm{C}=\mathrm{ONHCH}_{3}$

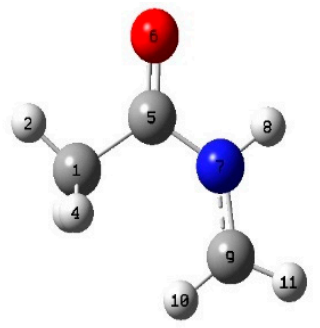

$\mathrm{CH}_{3}-\mathrm{C}=\mathrm{ONHC} \cdot \mathrm{H}_{2}$

Figure 2. Optimized structures of the radicals at B3LYP/6-31G $(d, p)$ level. 
3.3. Heats of Formation, Bond Energies, and Relative Stability of the Radicals Derived from the Target Parent Molecules

Four isodesmic reactions for each radical and the calculated standard enthalpies are listed in Table 3. The data illustrate excellent agreement across the different levels of calculations and through the different reaction analyses.

The enthalpies of formation of the parent molecules averaged over three work reactions for molecules $\mathrm{CH}_{3}-\mathrm{C}=\mathrm{ONHNH}_{2}, \mathrm{CH}_{3}-\mathrm{C}=\mathrm{ONH}_{2}$ and $\mathrm{CH}_{3}-\mathrm{C}=\mathrm{ONHCH}_{3}$ were evaluated as $-28.1,-57.29$, and $-56.53 \mathrm{kcal} \mathrm{mol}^{-1}$, respectively (values are average of B3LYP / 6-31g(d,p), B3LYP/6-31g(2d,2p) and CBS-QB3 levels).

\subsubsection{Enthalpy of Formation-Radicals}

The four work reactions and calculated $\Delta \mathrm{H}_{\mathrm{f}}{ }^{\circ} 298$ for use in determining the enthalpy of formation for each radical are shown in Table 3.

The recommended enthalpies of formation, in $\mathrm{kcal} \mathrm{mol}^{-1}$, from the CBS-QB3 level calculations, averaged over four work reactions are:

(i) Radicals from $\mathrm{CH}_{3}-\mathrm{C}=\mathrm{ONHNH}_{2}$ :
(a) $\mathrm{C} \bullet \mathrm{H}_{2}-\mathrm{C}=\mathrm{ONHNH}_{2}$ (19.27),
(b) $\mathrm{CH}_{3}-\mathrm{C}=\mathrm{ON} \bullet \mathrm{NH}_{2}(-2.07)$,
(c) $\mathrm{CH}_{3}-\mathrm{C}=\mathrm{ONHN} \bullet \mathrm{H}(1.60)$.

(ii) Radicals from $\mathrm{CH}_{3}-\mathrm{C}=\mathrm{ONH}_{2}$ :
a. $\mathrm{C} \bullet \mathrm{H}_{2}-\mathrm{C}=\mathrm{ONH}_{2}(-9.67)$,
b. $\mathrm{CH}_{3}-\mathrm{C}=\mathrm{ON} \bullet \mathrm{H}$ (2.11).

(iii) Radicals from $\mathrm{CH}_{3}-\mathrm{C}=\mathrm{ONHCH}_{3}$
a. $\mathrm{C} \bullet \mathrm{H}_{2}-\mathrm{C}=\mathrm{ONHCH}_{3}(-9.12)$,
b. $\mathrm{CH}_{3}-\mathrm{C}=\mathrm{ON} \bullet \mathrm{CH}_{3}(-4.43)$,
c. $\mathrm{CH}_{3}-\mathrm{C}=\mathrm{ONHC} \bullet \mathrm{H}_{2}(-15.39)$.

\subsubsection{Bond Energies}

Bond energies for the formation of radicals by loss of $\mathrm{H}$ atom reported at $298.15 \mathrm{~K}$ were calculated from the standard $\Delta \mathrm{H}_{\mathrm{f}}{ }^{\circ} 298$ values of the parent molecules and of the radicals, obtained at CBS-QB3 level. $\Delta \mathrm{H}_{\mathrm{f}}{ }^{\circ} 298$ of $52.1 \mathrm{kcal} \mathrm{mol}^{-1}$ was used for $\mathrm{H}$ atom enthalpy.

$$
\begin{gathered}
\mathrm{CH}_{3}-\mathrm{C}=\mathrm{ONHNH}_{2} \rightarrow \mathrm{C} \bullet \mathrm{H}_{2}-\mathrm{C}=\mathrm{ONHNH}_{2}+\mathrm{H} \bullet \\
-28.1419 .2752 .01 \mathrm{kcal} \mathrm{mol}^{-1} \\
\Delta \mathrm{Hrxn}=[19.27+52.1]-[-28.14]=99.51 \mathrm{kcal} \mathrm{mol}^{-1}=\text { Bond Energy }
\end{gathered}
$$

The bond dissociation enthalpies of the radicals calculated at three different levels of theory are listed in Table 3. The largest difference in $\mathrm{R}-\mathrm{H}$ bond energy for a given radical, considering the two DFT and the CBS-QB3 calculation methods and the four isodesmic reactions of each radical, was less than $1.5 \mathrm{kcal} \mathrm{mol}^{-1}$.

The bond dissociation energies for the $\mathrm{C}-\mathrm{H}$ bonds in the methyl group adjacent to the carbonyl in $\mathrm{CH}_{3}-\mathrm{C}=\mathrm{ONHNH}_{2}$ (99.51), $\mathrm{CH}_{3}-\mathrm{C}(=\mathrm{O}) \mathrm{NH}_{2}$ (99.72), and $\mathrm{CH}_{3}-\mathrm{C}=\mathrm{ONHCH}_{3}$ (99.51) $\mathrm{kcal} \mathrm{mol}^{-1}$ are in parentheses. These compare with the typical bond energy on a primary methyl site of a normal hydrocarbon of $101 \mathrm{kcal} \mathrm{mol}^{-1}$, and are ca. $2 \mathrm{kcal} \mathrm{mol}^{-1}$ lower. In contrast, they are ca. $3 \mathrm{kcal} \mathrm{mol}^{-1}$ higher than a typical primary methyl C-H bond on a ketone, which is $96 \mathrm{kcal} \mathrm{mol}^{-1}$. The $\mathrm{C}-\mathrm{H}$ bond energy on the methyl group bonded to the amine to form the $\mathrm{CH}_{3}-\mathrm{C}=\mathrm{ONHC} \bullet \mathrm{H}_{2}$ is $93.24 \mathrm{kcal} \mathrm{mol}^{-1}$. 
Table 3. Calculated standard enthalpies of formation $\left(\Delta \mathrm{H}_{\mathrm{f}}{ }^{\circ} 298\right)$ of radicals and $\mathrm{R}-\mathrm{H}$ bond energies.

\begin{tabular}{|c|c|c|c|}
\hline Radical $\mathrm{C}_{\bullet} \mathrm{H}_{2} \mathrm{CONHNH}_{2}$ Units: $\left(\mathrm{kcal} \mathrm{mol}^{-1}\right)$ & B3LYP/6-31G(d,p) & B3LYP6-31G(2d,2p) & CBS-QB3 \\
\hline $\mathrm{C} \bullet \mathrm{H}_{2} \mathrm{CONHNH}_{2}+\mathrm{CH}_{3} \mathrm{OH} \rightarrow \mathrm{CH}_{3} \mathrm{CONHNH}_{2}+\mathrm{C} \bullet \mathrm{H}_{2} \mathrm{OH}$ & 18.96 & 19.14 & 19.13 \\
\hline $\mathrm{C} \bullet \mathrm{H}_{2} \mathrm{CONHNH}_{2}+\mathrm{CH}_{3} \mathrm{NH}_{2} \rightarrow \mathrm{CH}_{3} \mathrm{CONHNH}_{2}+\mathrm{C} \bullet \mathrm{H}_{2} \mathrm{NH}_{2}$ & 18.14 & 18.09 & 18.76 \\
\hline${\mathrm{C} \bullet \mathrm{H}_{2} \mathrm{CONHNH}}_{2}+\mathrm{CH}_{3} \mathrm{CHO} \rightarrow \mathrm{CH}_{3} \mathrm{CONHNH}_{2}+\mathrm{C} \bullet \mathrm{H}_{2} \mathrm{CHO}$ & 19.94 & 19.78 & 19.94 \\
\hline $\mathrm{C} \bullet \mathrm{H}_{2} \mathrm{CONHNH}_{2}+\mathrm{CH}_{3} \mathrm{CH}_{2} \mathrm{CH}_{3} \rightarrow \mathrm{CH}_{3} \mathrm{CONHNH}_{2}+\mathrm{CH}_{3} \mathrm{C} \bullet \mathrm{HCH}_{3}$ & 20.02 & 19.63 & 19.67 \\
\hline Standard Enthalpy-Average & 19.26 & 19.16 & 19.37 \\
\hline Bond Energy $\mathrm{H}-\mathrm{CH}_{2} \mathrm{CONHNH}_{2}$ & 99.50 & 99.40 & 99.61 \\
\hline \multicolumn{4}{|l|}{ Radical $\mathrm{CH}_{3} \mathrm{CON} \bullet \mathrm{NH}_{2}$} \\
\hline $\mathrm{CH}_{3} \mathrm{CON} \bullet \mathrm{NH}_{2}+\mathrm{CH}_{3} \mathrm{NH}_{2} \rightarrow \mathrm{CH}_{3} \mathrm{CONHNH} H_{2}+\mathrm{CH}_{3} \mathrm{~N} \bullet \mathrm{H}$ & -0.32 & -0.06 & -0.52 \\
\hline $\mathrm{CH}_{3} \mathrm{CON} \bullet \mathrm{NH}_{2}+\mathrm{NH}_{2} \mathrm{NH}_{2} \rightarrow \mathrm{CH}_{3} \mathrm{CONHNH}{ }_{2}+\mathrm{NH}_{2} \mathrm{~N} \bullet \mathrm{H}$ & -2.39 & -2.71 & -2.68 \\
\hline $\mathrm{CH}_{3} \mathrm{CON} \bullet \mathrm{NH}_{2}+\mathrm{NH} 3 \rightarrow \mathrm{CH}_{3} \mathrm{CONHNH}_{2}+\mathrm{N} \bullet \mathrm{H}_{2}$ & -2.40 & -2.11 & -2.02 \\
\hline $\mathrm{CH}_{3} \mathrm{CON} \bullet \mathrm{NH}_{2}+\mathrm{CH}_{3} \mathrm{CH}_{2} \mathrm{OH} \rightarrow \mathrm{CH}_{3} \mathrm{CONHNH}_{2}+\mathrm{CH}_{3} \mathrm{C} \bullet \mathrm{HOH}$ & -3.93 & -3.24 & -2.41 \\
\hline Standard Enthalpy-Average & -2.26 & -2.03 & -1.91 \\
\hline Bond Energy $\mathrm{CH}_{3} \mathrm{CO}(\mathrm{N}-\mathrm{H}) \mathrm{NH}_{2}$ & 77.98 & 78.21 & 78.33 \\
\hline \multicolumn{4}{|l|}{ Radical $\mathrm{CH}_{3} \mathrm{CONHN \bullet H}$} \\
\hline $\mathrm{CH}_{3} \mathrm{CONHN} \bullet \mathrm{H}+\mathrm{CH}_{3} \mathrm{NH}_{2} \rightarrow \mathrm{CH}_{3} \mathrm{CONHNH}_{2}+\mathrm{CH}_{3} \mathrm{~N} \bullet \mathrm{H}$ & 3.63 & 3.18 & 3.28 \\
\hline $\mathrm{CH}_{3} \mathrm{CONHN} \bullet \mathrm{H}+\mathrm{NH}_{2} \mathrm{NH}_{2} \rightarrow \mathrm{CH}_{3} \mathrm{CONHNH}_{2}+\mathrm{NH}_{2} \mathrm{~N} \bullet \mathrm{H}$ & 1.56 & 0.53 & 1.12 \\
\hline $\mathrm{CH}_{3} \mathrm{CONHN} \bullet \mathrm{H}+\mathrm{NH} 3 \rightarrow \mathrm{CH}_{3} \mathrm{CONHNH}_{2}+\mathrm{N} \bullet \mathrm{H}_{2}$ & 1.55 & 1.12 & 1.78 \\
\hline $\mathrm{CH}_{3} \mathrm{CONHN} \bullet \mathrm{H}+\mathrm{CH}_{3} \mathrm{CH}_{2} \mathrm{OH} \rightarrow \mathrm{CH}_{3} \mathrm{CONHNH}_{2}+\mathrm{CH}_{3} \mathrm{C} \bullet \mathrm{HOH}$ & 0.02 & 0 & 1.40 \\
\hline Standard Enthalpy-Average & 1.69 & 1.21 & 1.89 \\
\hline Bond Energy $\mathrm{CH}_{3} \mathrm{CONHHNH}-\mathrm{H}$ & 81.93 & 81.45 & 82.13 \\
\hline \multicolumn{4}{|l|}{ 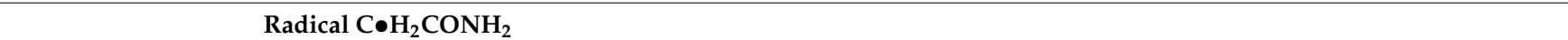 } \\
\hline $\mathrm{C} \bullet \mathrm{H}_{2} \mathrm{CONH}_{2}+\mathrm{CH}_{3} \mathrm{OH} \rightarrow \mathrm{CH}_{3} \mathrm{CONH}_{2}+\mathrm{C} \bullet \mathrm{H}_{2} \mathrm{OH}$ & -9.48 & -9.97 & -10.12 \\
\hline $\mathrm{C} \bullet \mathrm{H}_{2} \mathrm{CONH}_{2}+\mathrm{CH}_{3} \mathrm{NH}_{2} \rightarrow \mathrm{CH}_{3} \mathrm{CONH}_{2}+\mathrm{C} \bullet \mathrm{H}_{2} \mathrm{NH}_{2}$ & -10.29 & -11.03 & -10.49 \\
\hline $\mathrm{C} \bullet \mathrm{H}_{2} \mathrm{CONH}_{2}+\mathrm{CH}_{3} \mathrm{CHO} \rightarrow \mathrm{CH}_{3} \mathrm{CONH}_{2}+\mathrm{C} \bullet \mathrm{H}_{2} \mathrm{CHO}$ & -8.50 & -9.33 & -9.31 \\
\hline $\mathrm{C} \bullet \mathrm{H}_{2} \mathrm{CONH}_{2}+\mathrm{CH}_{3} \mathrm{CH}_{2} \mathrm{CH}_{3} \rightarrow \mathrm{CH}_{3} \mathrm{CONH}_{2}+\mathrm{CH}_{3} \mathrm{C} \bullet \mathrm{HCH}_{3}$ & -8.42 & -9.49 & -9.57 \\
\hline Standard Enthalpy-Average & -9.17 & -9.96 & -9.87 \\
\hline Bond Energy $\mathrm{H}-\mathrm{CH}_{2} \mathrm{CONH}_{2}$ & 100.21 & 99.43 & 99.52 \\
\hline \multicolumn{4}{|l|}{ Radical $\mathrm{CH}_{3} \mathrm{CON} \bullet \mathrm{H}$} \\
\hline $\mathrm{CH}_{3} \mathrm{CONH} \bullet+\mathrm{CH}_{3} \mathrm{NH}_{2} \rightarrow \mathrm{CH}_{3} \mathrm{CONH}_{2}+\mathrm{CH}_{3} \mathrm{~N} \bullet \mathrm{H}$ & 4.29 & 3.34 & 3.99 \\
\hline $\mathrm{CH}_{3} \mathrm{CONH} \bullet+\mathrm{NH}_{2} \mathrm{NH}_{2} \rightarrow \mathrm{CH}_{3} \mathrm{CONH}_{2}+\mathrm{NH}_{2} \mathrm{~N} \bullet \mathrm{H}$ & 2.22 & 0.69 & 1.83 \\
\hline $\mathrm{CH}_{3} \mathrm{CONH} \bullet+\mathrm{NH} 3 \rightarrow \mathrm{CH}_{3} \mathrm{CONH}_{2}+\mathrm{N} \bullet \mathrm{H}_{2}$ & 2.21 & 1.29 & 2.49 \\
\hline $\mathrm{CH}_{3} \mathrm{CONH} \bullet+\mathrm{CH}_{3} \mathrm{CH}_{2} \mathrm{OH} \rightarrow \mathrm{CH}_{3} \mathrm{CONH}_{2}+\mathrm{CH}_{3} \mathrm{C} \bullet \mathrm{HOH}$ & 0.68 & 0.16 & 2.10 \\
\hline Standard Enthalpy-Average & 2.35 & 1.37 & 2.60 \\
\hline Bond Energy $\mathrm{CH}_{3} \mathrm{CONH}-\mathrm{H}$ & 111.74 & 110.76 & 111.99 \\
\hline \multicolumn{4}{|l|}{ Radical $\mathrm{C}_{\bullet} \mathrm{H}_{2} \mathrm{CONHCH}_{3}$} \\
\hline $\mathrm{C} \bullet \mathrm{H}_{2} \mathrm{CONHCH}_{3}+\mathrm{CH}_{3} \mathrm{OH} \rightarrow \mathrm{CH}_{3} \mathrm{CONHCH}_{3}+\mathrm{C} \bullet \mathrm{H}_{2} \mathrm{OH}$ & -9.40 & -9.23 & -9.31 \\
\hline $\mathrm{C} \bullet \mathrm{H}_{2} \mathrm{CONHCH}_{3}+\mathrm{CH}_{3} \mathrm{NH}_{2} \rightarrow \mathrm{CH}_{3} \mathrm{CONHCH}_{3}+\mathrm{C} \bullet \mathrm{H}_{2} \mathrm{NH}_{2}$ & -10.21 & -10.29 & -9.69 \\
\hline $\mathrm{C} \bullet \mathrm{H}_{2} \mathrm{CONHCH}_{3}+\mathrm{CH}_{3} \mathrm{CHO} \rightarrow \mathrm{CH}_{3} \mathrm{CONHCH}_{3}+\mathrm{C}_{\bullet} \mathrm{H}_{2} \mathrm{CHO}$ & -8.42 & -8.59 & -8.51 \\
\hline $\mathrm{C} \bullet \mathrm{H}_{2} \mathrm{CONHCH}_{3}+\mathrm{CH}_{3} \mathrm{CH}_{2} \mathrm{CH}_{3} \rightarrow \mathrm{CH}_{3} \mathrm{CONHCH}_{3}+\mathrm{CH}_{3} \mathrm{C} \bullet \mathrm{HCH}_{3}$ & -8.34 & -8.74 & -8.77 \\
\hline Standard Enthalpy-Average & -9.10 & -9.21 & -9.07 \\
\hline Bond Energy $\mathrm{H}-\mathrm{CH}_{2} \mathrm{CONHCH}_{3}$ & 99.54 & 99.41 & 99.56 \\
\hline \multicolumn{4}{|l|}{ Radical $\mathrm{CH}_{3} \mathrm{CON} \bullet \mathrm{CH}_{3}$} \\
\hline $\mathrm{CH}_{3} \mathrm{CON} \bullet \mathrm{CH}_{3}+\mathrm{CH}_{3} \mathrm{NH}_{2} \rightarrow \mathrm{CH}_{3} \mathrm{CONHCH}_{3}+\mathrm{CH}_{3} \mathrm{~N} \bullet \mathrm{H}$ & -2.81 & -2.92 & -2.27 \\
\hline $\mathrm{CH}_{3} \mathrm{CON} \bullet \mathrm{CH}_{3}+\mathrm{NH}_{2} \mathrm{NH}_{2} \rightarrow \mathrm{CH}_{3} \mathrm{CONHCH}_{3}+\mathrm{NH}_{2} \mathrm{NHJ}$ & -4.88 & -5.57 & -4.43 \\
\hline $\mathrm{CH}_{3} \mathrm{CON} \bullet \mathrm{CH}_{3}+\mathrm{NH} 3 \rightarrow \mathrm{CH}_{3} \mathrm{CONHCH}_{3}+\mathrm{NH}_{2} \mathrm{~J}$ & -4.89 & -4.97 & -3.77 \\
\hline $\mathrm{CH}_{3} \mathrm{CON} \bullet \mathrm{CH}_{3}+\mathrm{CH}_{3} \mathrm{CH}_{2} \mathrm{OH} \rightarrow \mathrm{CH}_{3} \mathrm{CONHCH}_{3}+\mathrm{CH}_{3} \mathrm{C} \bullet \mathrm{HOH}$ & -6.42 & -6.10 & -4.15 \\
\hline Standard Enthalpy-Average & -4.75 & -4.89 & -3.65 \\
\hline Bond Energy $\mathrm{CH}_{3} \mathrm{CON}-\mathrm{HCH}_{3}$ & 103.88 & 103.74 & 104.98 \\
\hline \multicolumn{4}{|l|}{ Radical $\mathrm{CH}_{3} \mathrm{CONHC} \bullet \mathrm{H}_{2}$} \\
\hline $\mathrm{CH}_{3} \mathrm{CONHC} \bullet \mathrm{H}_{2}+\mathrm{CH}_{3} \mathrm{OH} \rightarrow \mathrm{CH}_{3} \mathrm{CONHCH}_{3}+\mathrm{C} \bullet \mathrm{H}_{2} \mathrm{OH}$ & -15.58 & -15.64 & -15.50 \\
\hline $\mathrm{CH}_{3} \mathrm{CONHC} \bullet \mathrm{H}_{2}+\mathrm{CH}_{3} \mathrm{NH}_{2} \rightarrow \mathrm{CH}_{3} \mathrm{CONHCH} 3+\mathrm{C} \bullet \mathrm{H}_{2} \mathrm{NH}_{2}$ & -16.39 & -16.70 & -15.88 \\
\hline $\mathrm{CH}_{3} \mathrm{CONHC} \bullet \mathrm{H}_{2}+\mathrm{CH}_{3} \mathrm{CHO} \rightarrow \mathrm{CH}_{3} \mathrm{CONHCH}_{3}+\mathrm{C} \bullet \mathrm{H}_{2} \mathrm{CHO}$ & -14.60 & -15 & -14.70 \\
\hline $\mathrm{CH}_{3} \mathrm{CONHC} \bullet \mathrm{H}_{2}+\mathrm{CH}_{3} \mathrm{CH}_{2} \mathrm{CH}_{3} \rightarrow \mathrm{CH}_{3} \mathrm{CONHCH}_{3}+\mathrm{CH}_{3} \mathrm{C} \bullet \mathrm{HCH}_{3}$ & -14.52 & -15.15 & -14.96 \\
\hline Standard Enthalpy-Average & -15.27 & -15.62 & -15.26 \\
\hline Bond Energy $\mathrm{CH}_{3} \mathrm{CONHCH}_{2}-\mathrm{H}$ & 93.36 & 93.01 & 93.37 \\
\hline
\end{tabular}

The $\mathrm{N}-\mathrm{H}$ bond strengths for nitrogen atom adjacent to the carbonyl groups in $\mathrm{CH}_{3}-$ $\mathrm{C}=\mathrm{ONHNH}_{2}$ (78.17), $\mathrm{CH}_{3}-\mathrm{C}(=\mathrm{O}) \mathrm{NH}_{2}$ (111.50), and $\mathrm{CH}_{3}-\mathrm{C}=\mathrm{ONHCH}_{3}$ (104.19) $\mathrm{kcal} \mathrm{mol}^{-1}$ are in the parenthesis. For comparison, the $\mathrm{N}-\mathrm{H}$ bond in ammonia is (108), the $\mathrm{CH}_{3} \mathrm{NH}-\mathrm{H}$ bond in methylamine is (102.4), and the $\mathrm{NH}_{2} \mathrm{NH}-\mathrm{H}$ bond in hydrazine is (82) $\mathrm{kcal} \mathrm{mol}^{-1}$.

The $\mathrm{C}-\mathrm{H}$ bond strength in the $\mathrm{CH}_{3}$ group bonded to an amine in $\mathrm{CH}_{3}-\mathrm{C}=\mathrm{ONHCH}_{2}-\mathrm{H}$ is $93.37 \mathrm{kcal} \mathrm{mol}^{-1}$ at the CBS-QB3 level. This compares with the $\mathrm{C}-\mathrm{H}$ bond energy of 
$\mathrm{H}-\mathrm{CH}_{2}-\mathrm{NH}_{2}(\sim 94.4)$ in $\mathrm{CH}_{3} \mathrm{NH}_{2}$. This $\mathrm{C}-\mathrm{H}$ bond in $\mathrm{CH}_{3}-\mathrm{C}=\mathrm{ONHC} \bullet \mathrm{H}_{2}$ is $1 \mathrm{kcal} \mathrm{mol}^{-1}$ lower than on the methyl groups bonded to $\mathrm{C}=\mathrm{O}$.

The $\mathrm{N}-\mathrm{H}$ bond of the N.H. group in $\mathrm{CH}_{3}-\mathrm{C}=\mathrm{O}-\mathrm{N} \bullet-\mathrm{NH}_{2}$ is the weakest in this molecule at $78.17 \mathrm{kcal} \mathrm{mol}^{-1}$, where the $\mathrm{C}-\mathrm{H}$ bond in $\mathrm{C} \bullet \mathrm{H}_{2}-\mathrm{C}=\mathrm{ONHNH}_{2}$ is $99.51 \mathrm{kcal} \mathrm{mol}^{-1}$, which is significantly higher. The $\mathrm{N}-\mathrm{H}$ bond energy for acetohydrazide $\left(\mathrm{CH}_{3}-\mathrm{C}=\mathrm{ONHNH} \bullet\right)$ is markedly different at only $81.84 \mathrm{kcal} \mathrm{mol}^{-1}$. In the $\mathrm{N}-\mathrm{H}$ bond cleavage of $\mathrm{CH}_{3}-$ $\mathrm{C}=\mathrm{ON} \bullet \mathrm{NH}_{2}$, the electrons from the radical site re-arrange to form a second double bond, from nitrogen to carbon. The $\mathrm{N}-\mathrm{H}$ bond energy for $\mathrm{CH}_{3}-\mathrm{C}=\mathrm{ON} \bullet \mathrm{CH}_{3}$ is $104.20 \mathrm{kcal} \mathrm{mol}^{-1}$ which is much higher than the $\mathrm{CH}_{3}-\mathrm{C}=\mathrm{ON} \bullet \mathrm{NH}_{2}$.

The weakening of the $\mathrm{N}-\mathrm{H}$ bonds in this hydrazide is essentially independent of the nature of the $\beta$-substituent $\left(\mathrm{H}, \mathrm{RCO}, \mathrm{CO}_{2} \mathrm{Et}\right.$, or $\left.\mathrm{PhSO}_{2}\right)$ and the stabilizing effect on the radical is brought about entirely by the three-electron on $\mathrm{N}-\mathrm{NH}_{2}$ moiety [27].

\subsection{Internal Rotor Potentials}

Energy profiles for internal rotations about the $\mathrm{C}-\mathrm{C}=\mathrm{O}, \mathrm{O}=\mathrm{C}-\mathrm{N}, \mathrm{N}-\mathrm{C}$, and $\mathrm{N}-\mathrm{N}$ bonds in the acetohydrazide, acetamide, and $\mathrm{N}$-methyl acetamide were calculated to determine the lowest energy configurations, energies of the rotational conformers, and to identify the interconversion barriers between isomers. Torsional potentials were used to evaluate contributions to the entropy and heat capacity values when there were low barriers (less than $3.5 \mathrm{kcal} \mathrm{mol}^{-1}$ ) and internal rotation occurred.

The total energies as a function of the corresponding dihedral angles were computed at the B3LYP $/ 6-31 \mathrm{G}(\mathrm{d}, \mathrm{p})$ level of theory by scanning the torsion angles between $0^{\circ}$ and $360^{\circ}$ in steps of $15^{\circ}$, while all remaining coordinates were fully optimized. All potentials were rescanned when a lower energy conformer, relative to the initial low-energy conformer was found. The total energy of the corresponding most stable molecular conformer was arbitrarily set to zero and used as a reference point to plot the potential barriers. The resulting potential energy barriers for internal rotations in the stable nonradical and radical molecules are shown in Figures 3-9. Dihedral angles obtained for the optimized lowest energy structures are shown in parentheses.

Potential energy barriers for internal rotations about $\mathrm{N}-\mathrm{N}$ and $\mathrm{O}=\mathrm{C}-\mathrm{N}$ bond in $\mathrm{CH}_{3}-\mathrm{C}=\mathrm{O}-\mathrm{NH}-\mathrm{NH}_{2}$ and in $\mathrm{CH}_{3}-\mathrm{C}=\mathrm{O}-\mathrm{NH}^{-\mathrm{CH}_{3}}$

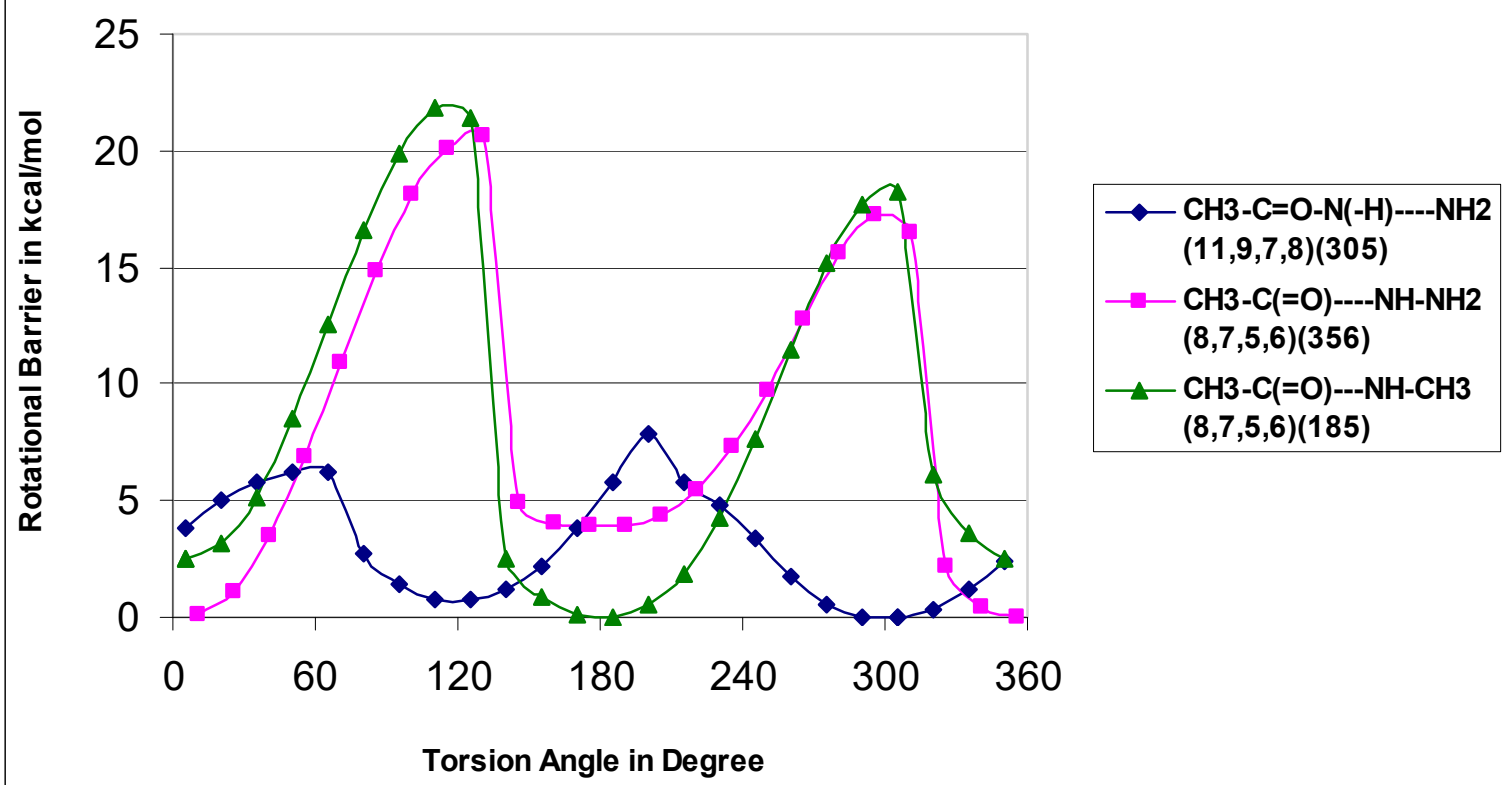

Figure 3. Potential energy barriers for internal rotations about $\mathrm{N}-\mathrm{N}$ and $\mathrm{O}=\mathrm{C}-\mathrm{N}$ bonds in $\mathrm{CH}_{3}-\mathrm{C}=\mathrm{O}-\mathrm{NH}-\mathrm{NH} \mathrm{H}_{2}$ and $\mathrm{CH}_{3}-\mathrm{C}=\mathrm{O}-\mathrm{NH}-\mathrm{CH}_{3}$. 
The $\mathrm{CH}_{3}-\mathrm{C}=\mathrm{O}-\mathrm{NH}-\mathrm{NH}_{2}$ rotor is illustrated in Figure 3 for both the $\mathrm{CH}_{3}-\mathrm{C}=\mathrm{O}-\mathrm{N}(-$ $\mathrm{H})-\mathrm{NH}_{2}(\mathrm{H} 10-\mathrm{N} 9-\mathrm{N} 7-\mathrm{H} 8)$ and $\mathrm{CH}_{3}-\mathrm{C}(=\mathrm{O})-\mathrm{NH}-\mathrm{NH}_{2}(\mathrm{H} 8-\mathrm{N} 7-\mathrm{C} 5-\mathrm{O} 6)$ systems with barriers at 7.82 and $20.66 \mathrm{kcal} \mathrm{mol}^{-1}$, respectively. The $\mathrm{CH}_{3}-\mathrm{C}(=\mathrm{O})-\mathrm{NH}-\mathrm{CH}_{3}(\mathrm{H} 8-\mathrm{N} 7-$ C5-O6) rotor shows a two-fold symmetry with a barrier at $21.7 \mathrm{kcal} \mathrm{mol}^{-1}$. One reason for the high barriers for the rotation about the carbonyl $(\mathrm{C}=\mathrm{O})-\mathrm{NH}$ bond involves the repulsive interaction of the carbonyl $\pi$ bond with the $\mathrm{NH}$ lone pair.

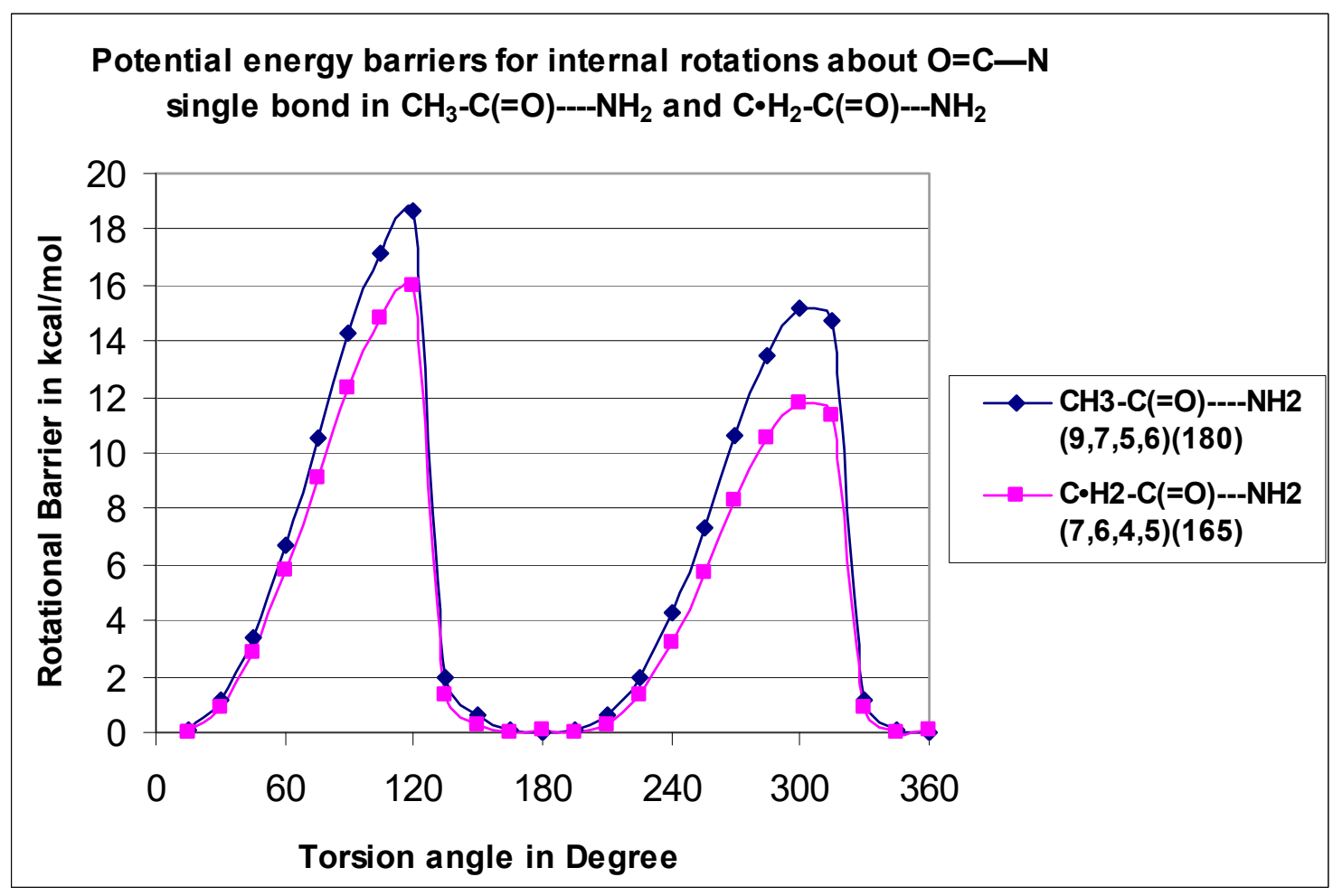

Figure 4. Potential energy barriers for internal rotations about $\mathrm{O}=\mathrm{C}-\mathrm{N}$ single bond in acetamide.

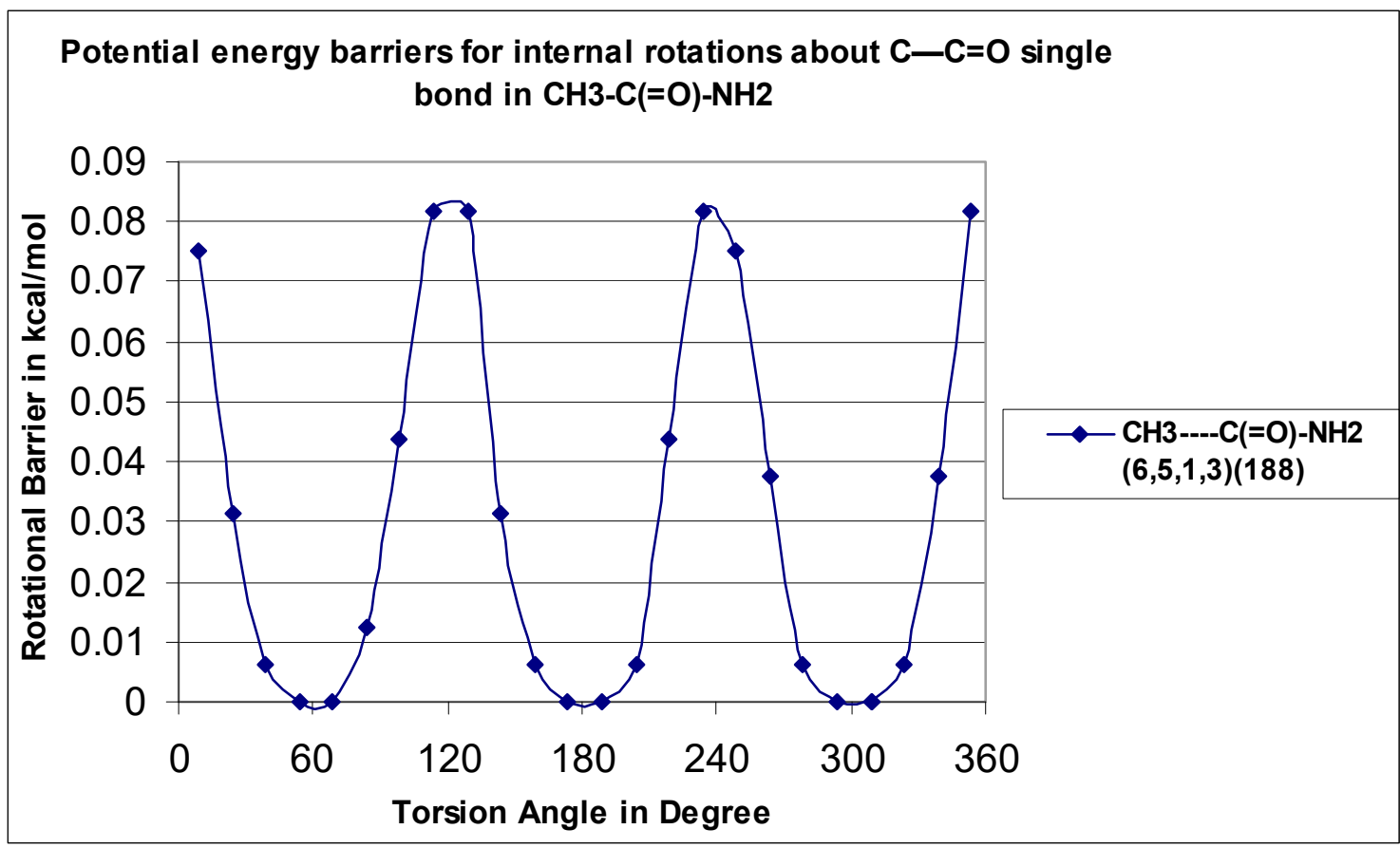

Figure 5. Potential energy barriers for internal rotations about $\mathrm{C}-\mathrm{C}=\mathrm{O}$ single bond in acetamide. 


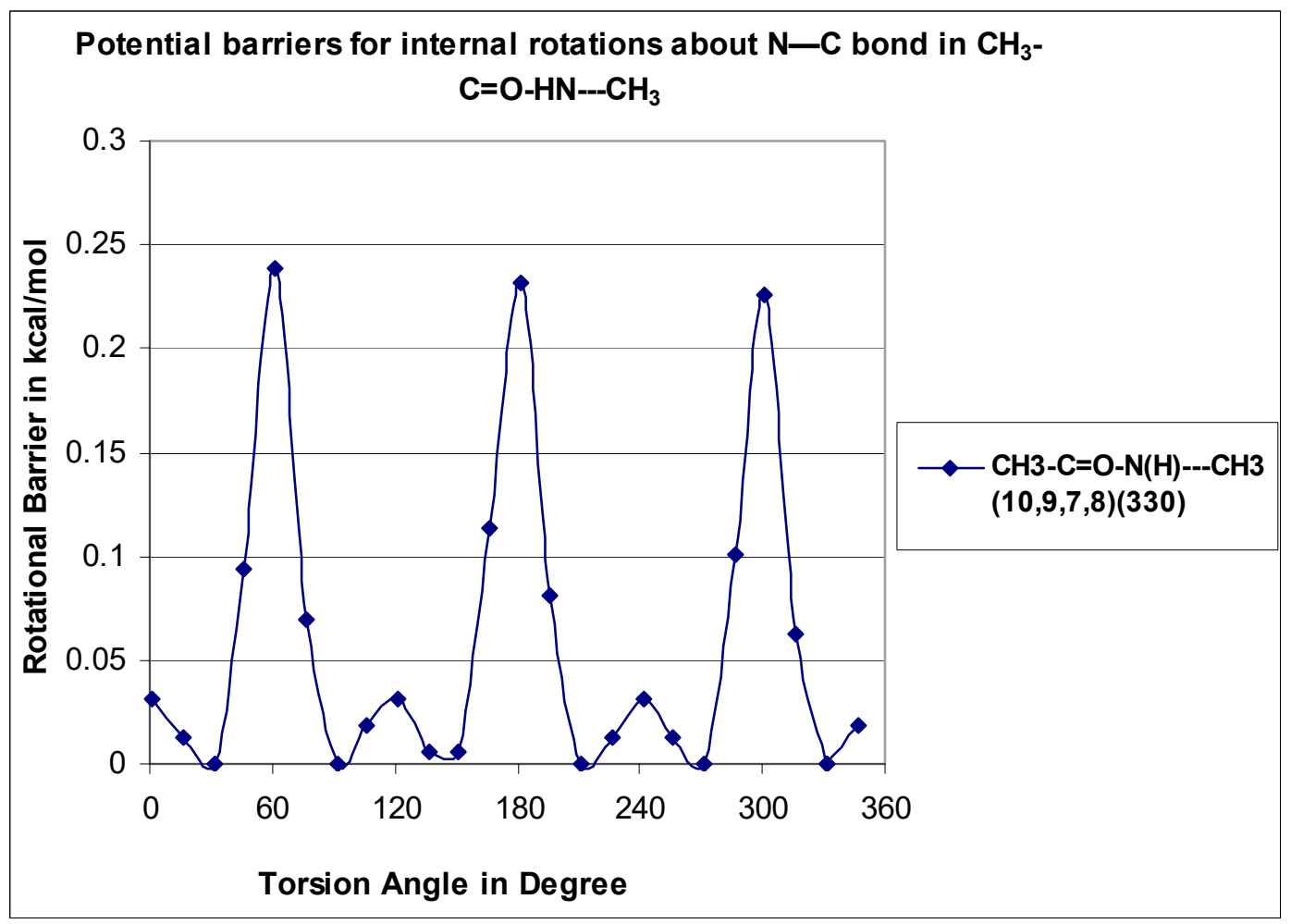

Figure 6. Potential barriers for internal rotations about $\mathrm{N}-\mathrm{C}$ bond in $\mathrm{N}$-methyl acetamide.

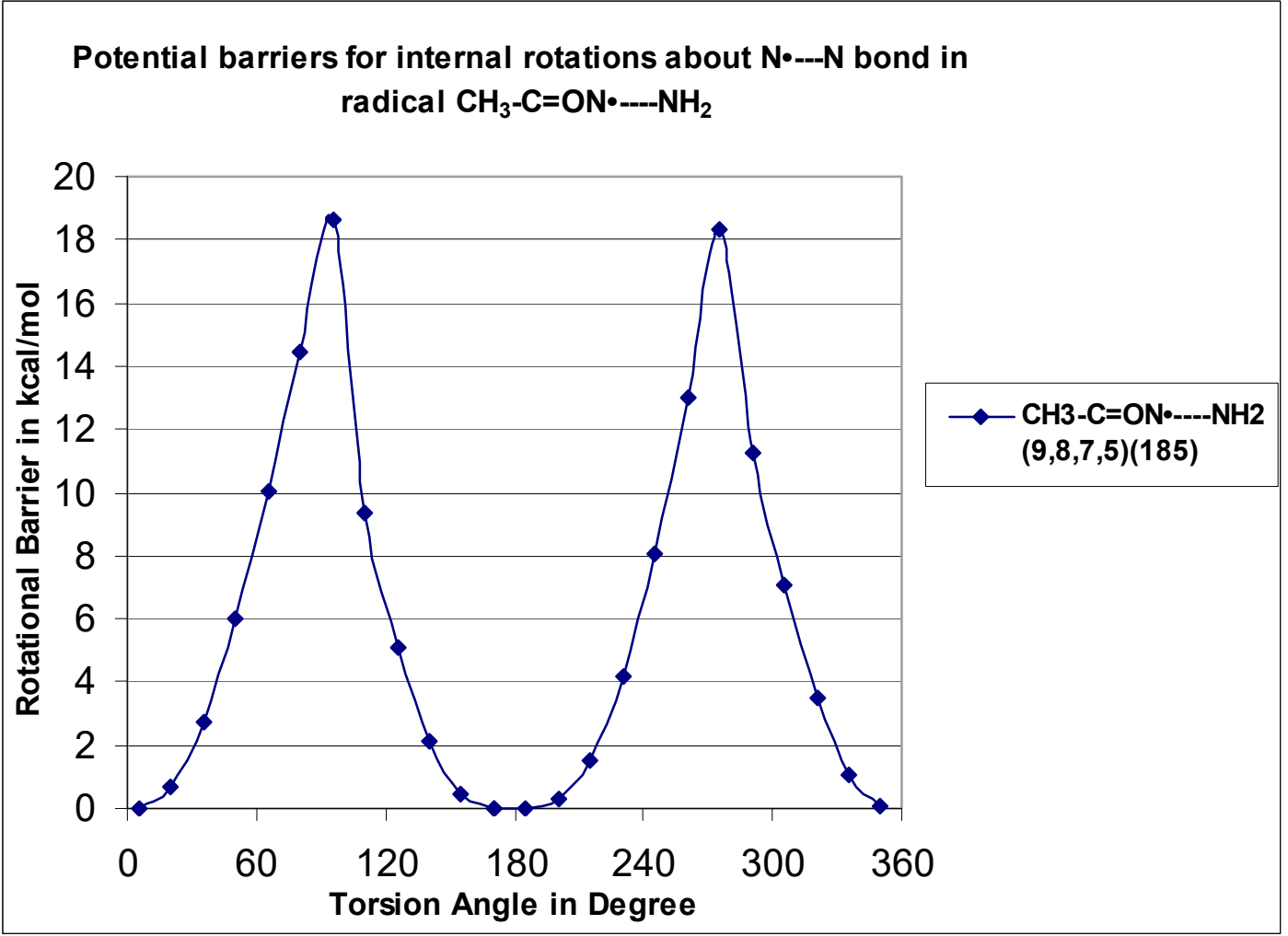

Figure 7. Potential barriers for internal rotations about $\mathrm{N} \bullet-\mathrm{N}$ bond in radical $\mathrm{CH}_{3}-\mathrm{C}=\mathrm{ON} \bullet-\mathrm{NH}_{2}$ which shows a two-fold symmetry with a barrier at $14.6 \mathrm{kcal} \mathrm{mol}^{-1}$. 


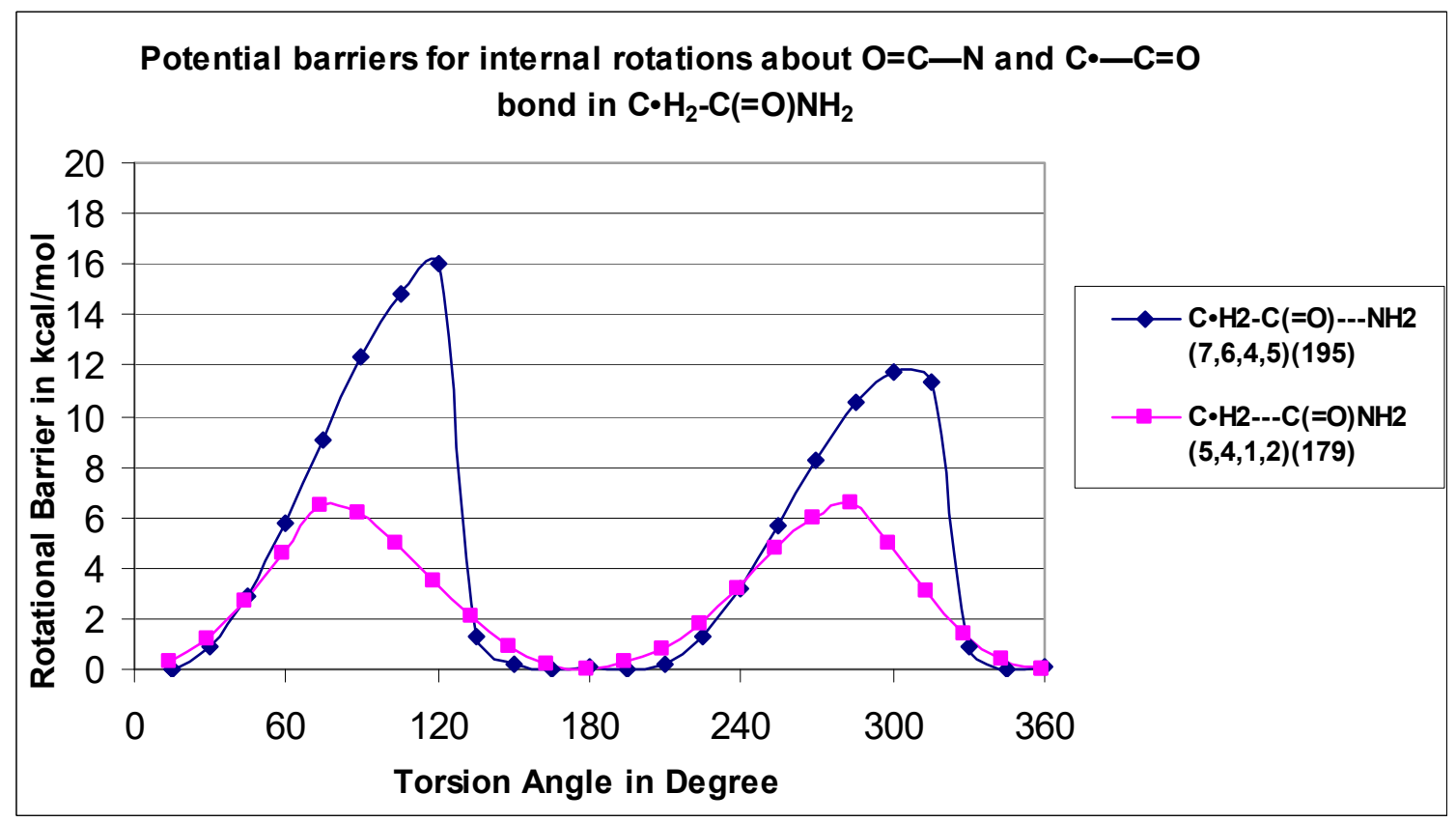

Figure 8. Potential barriers for internal rotations about $\mathrm{O}=\mathrm{C}-\mathrm{N}$ and $\mathrm{C} \bullet-\mathrm{C}=\mathrm{O}$ bond in acetamide radical $\mathrm{C} \bullet \mathrm{H}_{2}-\mathrm{C}(=\mathrm{O}) \mathrm{NH}$.

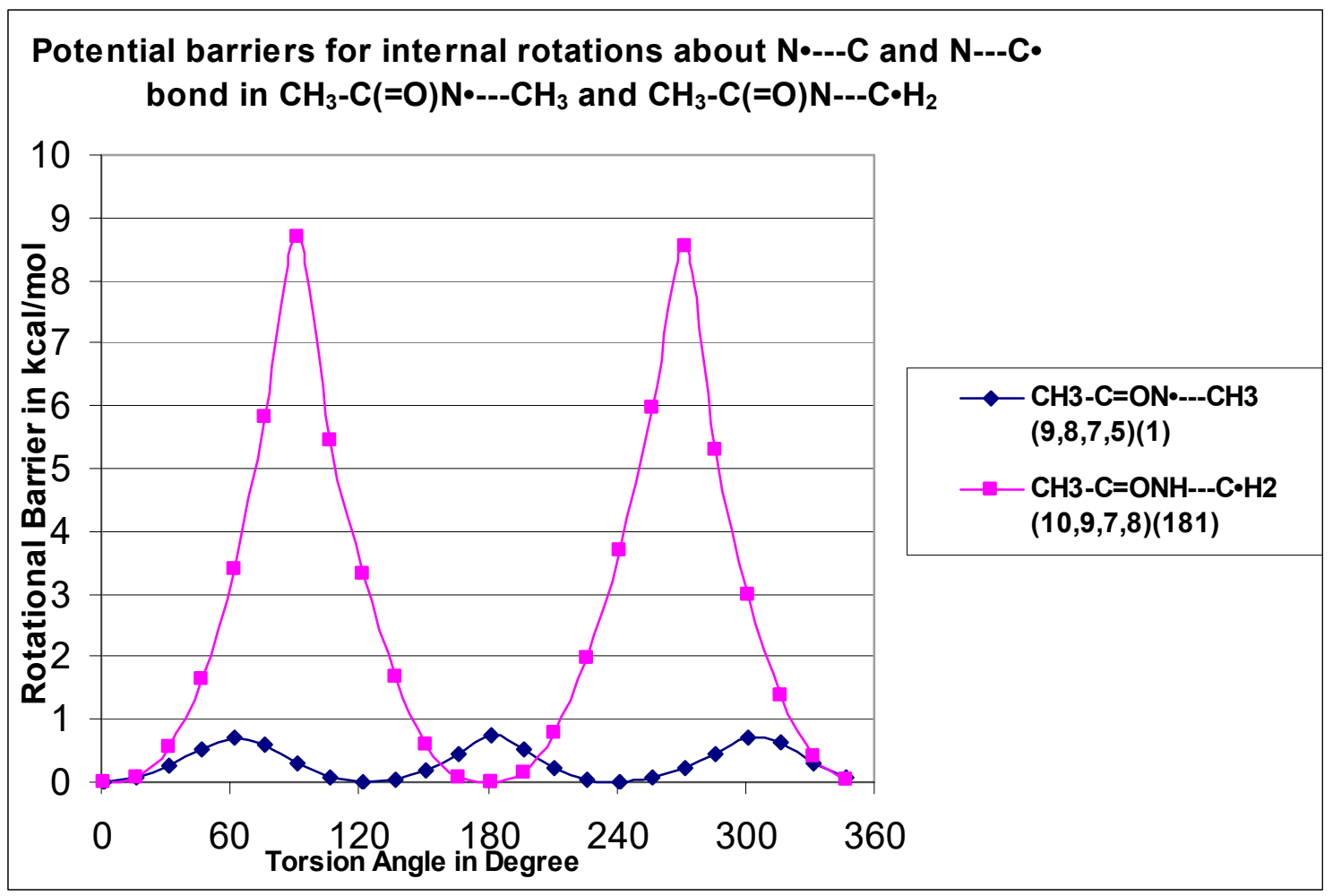

Figure 9. Potential barriers for internal rotations about $\mathrm{N} \bullet-\mathrm{C}$ and $\mathrm{N}-\mathrm{C} \bullet$ bond in $\mathrm{CH}_{3}-\mathrm{C}(=\mathrm{O}) \mathrm{N} \bullet-\mathrm{CH}_{3}$ and $\mathrm{CH}{ }_{3}-\mathrm{C}(=\mathrm{O}) \mathrm{N}-$ $\mathrm{C} \bullet \mathrm{H}_{2}$.

The study of amide $\mathrm{C}-\mathrm{N}$ rotation barriers is important for the evaluation of their reactivity, for input data in biochemical structure calculations other than finding the lowest energy conformer because the amide $\mathrm{C}-\mathrm{N}$ bonds make up protein backbones. The amide conformations play an important role in enzyme structure and the barrier to the rotation affects the rigidity of that structure. The rigidity of an enzyme's structure can affect its selectivity in binding substrates. 
The $\mathrm{C}-\mathrm{N}$ rotor for the radical $\mathrm{C} \bullet \mathrm{H}_{2}-\mathrm{C}(=\mathrm{O})-\mathrm{NH}_{2}\left(\mathrm{H}_{7}-\mathrm{N}_{6}-\mathrm{C}_{4}-\mathrm{O}_{5}\right.$ is $\left.15.97 \mathrm{kcal} \mathrm{mol}^{-1}\right)$ and the value of its parent $\mathrm{CH}_{3}-\mathrm{C}(=\mathrm{O})-\mathrm{NH}_{2}\left(\mathrm{H}_{8}-\mathrm{N}_{7}-\mathrm{C}_{5}-\mathrm{O}_{6}\right.$ is $\left.18.66 \mathrm{kcal} \mathrm{mol}^{-1}\right)$. According to Jasien et al. [12], based upon the current $(\mathbf{D Z}+\mathbf{d} / \mathbf{S C F})$ calculations, the $0 \mathrm{~K}$ rotational barrier for acetamide between the $\mathrm{C}-\mathrm{N}$ bond is predicted to be $12.5 \mathrm{kcal} \mathrm{mol}^{-1}$.

The two protons attached to $\mathrm{N}$ in acetamide are inequivalent at low temperatures but become averaged by $\mathrm{C}-\mathrm{N}$ rotation at higher temperatures. Typical bond rotation barriers for amides in solution are experimentally determined by NMR to be between 17 and $22 \mathrm{kcal} / \mathrm{mol}$. However, the acetamide enolate, [CH2CONH2] has a much lower barrier; according to NMR experiments, the enolate has free rotation at all accessible temperatures [28]. The HNCO anti/trans/ $Z$ configuration is significantly more probable in proteins than the $s y n / c i s / E$ geometry. The ratio is $95: 5$ or even higher [29].

The $\mathrm{CH}_{3}-\mathrm{C}=\mathrm{O}$ rotor for the $\mathrm{CH}_{3}-\mathrm{C}(=\mathrm{O})-\mathrm{NH}_{2}\left(\mathrm{O}_{6}-\mathrm{C}_{5}-\mathrm{C}_{1}-\mathrm{H}_{2}\right)$ has a small barrier at $0.08 \mathrm{kcal} \mathrm{mol}^{-1}$ and shows three-fold symmetry The data are in reasonable agreement with the previous study conducted by J.R. Bailey [30], which also reported a low, calculated barrier monosubstituted $N$-methyl acetamide to be $0.21 \mathrm{kcal} \mathrm{mol}^{-1}$.

The $\mathrm{CH}_{3}-\mathrm{C}=\mathrm{O}-\mathrm{HN}-\mathrm{CH}_{3}$ rotor is illustrated in Figure 6 which shows a three-fold symmetry with a barrier at $0.24 \mathrm{kcal} \mathrm{mol}^{-1}$.

The $\mathrm{C}(=\mathrm{O}) \mathrm{NH}_{2}$ rotor is illustrated in Figure 8 for both the $\mathrm{C} \bullet \mathrm{H}_{2}-\mathrm{C}(=\mathrm{O})-\mathrm{NH}_{2}(\mathrm{H} 7-$ $\mathrm{N} 6-\mathrm{C} 4-\mathrm{O} 5)$ and $\mathrm{C} \bullet \mathrm{H} 2-\mathrm{C}(=\mathrm{O}) \mathrm{NH}_{2}(\mathrm{O} 5-\mathrm{C} 4-\mathrm{C} 1-\mathrm{H} 2)$ systems with a great difference in bariers at 16.0 and $6.6 \mathrm{kcal} \mathrm{mol}^{-1}$, respectively, but with the same two-fold symmetry.

The $\mathrm{CH}_{3}-\mathrm{C}(=\mathrm{O}) \mathrm{N} \bullet-\mathrm{CH}_{3}\left(\mathrm{H} 9-\mathrm{C} 8-\mathrm{N}_{7}-\mathrm{C} 5\right)$ and $\mathrm{CH}_{3}-\mathrm{C}(=\mathrm{O}) \mathrm{N}-\mathrm{C}_{\bullet} \mathrm{H}_{2}(\mathrm{H} 10-\mathrm{C} 9-\mathrm{N} 7-$ $\mathrm{H} 8$ ) rotors are illustrated in Figure 9 and there is a significant difference in the internal rotor barriers. $\mathrm{CH}_{3}-\mathrm{C}(=\mathrm{O}) \mathrm{N} \bullet-\mathrm{CH}_{3}$ shows extremely low three-fold symmetry at $0.7 \mathrm{kcal} \mathrm{mol}^{-1}$, and $\mathrm{CH}_{3}-\mathrm{C}(=\mathrm{O}) \mathrm{N}-\mathrm{C} \bullet \mathrm{H}_{2}$ shows two-fold symmetry at $8.7 \mathrm{kcal} \mathrm{mol}^{-1}$ barrier. The low barrier in $\mathrm{CH}_{3}-\mathrm{C}(=\mathrm{O}) \mathrm{N} \bullet-\mathrm{CH}_{3}$ is a result of the overlap between the carbonyl bond and the unpaired electron of the $\mathrm{N}$ atom, which reduces the interaction of the methyl $\mathrm{H}$ atoms with the $\mathrm{N}$ nitrogen $\pi$ orbitals. The higher barrier for $\mathrm{CH}_{3}-\mathrm{C}(=\mathrm{O}) \mathrm{N}-\mathrm{C}_{\bullet} \mathrm{H}_{2}$ results from overlap (resonance) between the radical and the nitrogen $\pi$ bonds.

\subsection{Entropy and Heat Capacity}

The entropy and heat capacity data for the parent molecules and their radicals as a function of temperature were determined from the optimized structures, moments of inertia, vibrational frequencies, symmetries, the known mass of the molecules, and internal rotor contributions when barriers were low. The calculations use standard formulas from statistical mechanics for the contributions of translation, external rotation, and vibrations [31,32]. Contributions to the entropy and the heat capacity from translation, vibrations, and external rotation were calculated using the SMCPS program. This program utilizes the rigid-harmonic oscillator approximation from the optimized structures obtained at B3LYP/6-31G $(\mathrm{d}, \mathrm{p})$ level. The number of optical isomers and the spin degeneracy of unpaired electrons is also incorporated for the calculation of $S^{\circ} 298$.

Contributions from hindered internal rotors to $S^{\circ} 298$ and $C p(T)$ are determined using the VIBIR program. This program utilizes the method of Pitzer and Gwinn [33,34], the potential barriers, folds, and moments of inertia from the internal rotor analysis. The moments of inertia were calculated. The rotors with a barrier value greater than $3.5 \mathrm{kcal} \mathrm{mol}^{-1}$ were treated as torsion vibrations. The internal rotor data were combined with the $S(T)$ and $\mathrm{Cp}(\mathrm{T})$ data from frequencies, mass, moments of inertia, symmetry, and electronic degeneracy in the our statistical mechanics program SMCPS [35] and are presented in Table 4 for the parent molecules, Table 5 for radicals from acetic acid hydrazide, Table 6 for radicals from acetamide, and Table 7 for radicals from $\mathrm{N}$-methyl acetamide. 
Table 4. Ideal gas-phase thermodynamic property vs. temperature ${ }^{\mathrm{a}}$ of parent molecules.

\begin{tabular}{|c|c|c|c|c|c|c|c|c|c|}
\hline \multirow{2}{*}{$\mathrm{T}(\mathrm{K})$} & \multirow{2}{*}{$\begin{array}{c}\Delta \mathrm{H}_{\mathrm{f}}{ }^{\circ} 298 \\
\text { kcal mol }^{-1}\end{array}$} & \multicolumn{2}{|c|}{$\mathrm{CH}_{3} \mathrm{CONHNH}_{2}$} & \multirow{2}{*}{$\begin{array}{c}\Delta \mathrm{H}_{\mathrm{f}}^{\circ} 298 \\
\mathrm{kcal} \mathrm{mol}^{-1}\end{array}$} & \multicolumn{2}{|c|}{$\mathrm{CH}_{3} \mathrm{CONH}_{2}$} & \multirow{2}{*}{$\begin{array}{c}\Delta \mathrm{H}_{\mathrm{f}}{ }^{\circ} 298 \\
\mathrm{kcal} \mathrm{mol}^{-1}\end{array}$} & \multicolumn{2}{|c|}{$\mathrm{CH}_{3} \mathrm{CONHCH}_{3}$} \\
\hline & & $\mathrm{Cp}(\mathrm{T})$ & $S^{\circ}(T)$ & & $\mathrm{Cp}(\mathrm{T})$ & $\mathrm{S}^{\circ}(\mathrm{T})$ & & $\mathrm{Cp}(\mathrm{T})$ & $S^{\circ}(T)$ \\
\hline 1 & \multirow{26}{*}{-28.58} & 7.949 & 16.841 & \multirow{26}{*}{-58.14} & 7.949 & 14.636 & \multirow{26}{*}{-56.54} & 7.949 & 14.677 \\
\hline 51 & & 8.877 & 48.268 & & 8.831 & 46.039 & & 8.532 & 45.934 \\
\hline 101 & & 10.851 & 54.999 & & 9.835 & 52.512 & & 10.155 & 52.332 \\
\hline 151 & & 13.154 & 59.82 & & 11.161 & 56.729 & & 12.071 & 56.795 \\
\hline 201 & & 15.609 & 63.932 & & 12.866 & 60.163 & & 14.237 & 60.555 \\
\hline 251 & & 18.098 & 67.676 & & 14.719 & 63.227 & & 16.57 & 63.973 \\
\hline 298 & & 21.575 & 71.052 & & 17.539 & 65.962 & & 21.065 & 67.078 \\
\hline 400 & & 26.417 & 77.763 & & 21.311 & 71.357 & & 26.215 & 73.371 \\
\hline 500 & & 30.648 & 83.881 & & 24.613 & 76.245 & & 30.961 & 79.271 \\
\hline 600 & & 34.249 & 89.6 & & 27.434 & 80.8 & & 35.118 & 84.912 \\
\hline 700 & & 36.275 & 94.949 & & 28.832 & 85.054 & & 36.677 & 90.28 \\
\hline 800 & & 39.881 & 99.96 & & 31.88 & 89.036 & & 41.778 & 95.376 \\
\hline 900 & & 41.108 & 104.665 & & 32.663 & 92.774 & & 42.417 & 100.209 \\
\hline 1000 & & 44.052 & 109.094 & & 35.21 & 96.294 & & 46.735 & 104.794 \\
\hline 1100 & & 44.75 & 113.275 & & 35.576 & 99.616 & & 46.733 & 109.148 \\
\hline 1200 & & 46.236 & 117.23 & & 36.772 & 102.761 & & 48.481 & 113.287 \\
\hline 1300 & & 47.54 & 120.98 & & 37.824 & 105.744 & & 50.005 & 117.225 \\
\hline 1400 & & 48.686 & 124.543 & & 38.751 & 108.579 & & 51.337 & 120.977 \\
\hline 1500 & & 50.695 & 127.934 & & 40.562 & 111.279 & & 54.499 & 124.556 \\
\hline 2000 & & 53.236 & 142.757 & & 42.451 & 123.09 & & 56.539 & 140.262 \\
\hline 2500 & & 55.241 & 154.866 & & 44.091 & 132.751 & & 58.785 & 153.137 \\
\hline 3000 & & 56.455 & 165.05 & & 45.088 & 140.882 & & 60.132 & 163.98 \\
\hline 3500 & & 57.235 & 173.814 & & 45.73 & 147.883 & & 60.992 & 173.316 \\
\hline 4000 & & 57.763 & 181.492 & & 46.164 & 154.018 & & 61.572 & 181.499 \\
\hline 4500 & & 58.135 & 188.317 & & 46.471 & 159.473 & & 61.979 & 188.775 \\
\hline 5000 & & 58.407 & 194.456 & & 46.695 & 164.381 & & 62.276 & 195.321 \\
\hline
\end{tabular}

a Thermodynamic properties refer to the standard state of an ideal gas at 1 atm. $\mathrm{S}^{\circ}(\mathrm{T})$ and $\mathrm{C}^{\circ} \mathrm{p}(\mathrm{T})$ in cal $\mathrm{mol}^{-1} \mathrm{~K}^{-1}$.

Table 5. Ideal gas-phase thermodynamic property vs. temperature a of radicals of parent $\mathrm{CH} 3 \mathrm{CONHNH} 2$.

\begin{tabular}{|c|c|c|c|c|c|c|c|c|c|}
\hline \multirow{2}{*}{$\mathbf{T}(\mathbf{K})$} & \multirow{2}{*}{$\begin{array}{c}\Delta \mathrm{H}_{\mathrm{f}}^{\circ} 298 \\
\mathrm{kcal} \mathrm{mol}^{-1}\end{array}$} & \multicolumn{2}{|c|}{$\mathrm{C} \cdot \mathrm{H}_{2}-\mathrm{C}=\mathrm{ONHNH}_{2}$} & \multirow{2}{*}{$\begin{array}{c}\Delta \mathrm{H}_{\mathrm{f}}{ }^{\circ} 298 \\
\mathrm{kcal} \mathrm{mol}^{-1}\end{array}$} & \multicolumn{2}{|c|}{$\mathrm{CH}_{3}-\mathrm{C}=\mathrm{ON} \cdot \mathrm{NH}_{2}$} & \multirow{2}{*}{$\begin{array}{c}\Delta \mathrm{H}_{\mathrm{f}}{ }^{\circ} 298 \\
\mathrm{kcal} \mathrm{mol}^{-1}\end{array}$} & \multicolumn{2}{|c|}{$\mathrm{CH}_{3}-\mathrm{C}=\mathrm{ONHN} \cdot \mathrm{H}$} \\
\hline & & $\mathrm{Cp}(\mathrm{T})$ & $S^{\circ}(T)$ & & $\mathrm{Cp}(\mathrm{T})$ & $S^{\circ}(\mathrm{T})$ & & $\mathrm{Cp}(\mathrm{T})$ & $\mathbf{S}^{\circ}(\mathrm{T})$ \\
\hline 1 & \multirow{26}{*}{19.11} & 7.949 & 20.227 & \multirow{26}{*}{-2.08} & 7.949 & 17.901 & \multirow{26}{*}{1.58} & 7.949 & 18.037 \\
\hline 51 & & 9.561 & 52.378 & & 8.899 & 49.318 & & 8.197 & 49.18 \\
\hline 101 & & 11.599 & 59.551 & & 10.976 & 56.109 & & 9.838 & 55.307 \\
\hline 151 & & 14.394 & 64.765 & & 13.16 & 60.965 & & 12.072 & 59.701 \\
\hline 201 & & 17.265 & 69.294 & & 15.366 & 65.047 & & 14.494 & 63.498 \\
\hline 251 & & 20 & 73.436 & & 17.568 & 68.707 & & 16.912 & 66.986 \\
\hline 298 & & 22.457 & 77.155 & & 21.532 & 71.967 & & 20.276 & 70.145 \\
\hline 400 & & 27.093 & 84.422 & & 25.642 & 78.367 & & 24.789 & 76.43 \\
\hline 500 & & 30.849 & 90.876 & & 29.268 & 84.138 & & 28.661 & 82.147 \\
\hline 600 & & 33.906 & 96.772 & & 32.372 & 89.496 & & 31.919 & 87.474 \\
\hline 700 & & 36.419 & 102.187 & & 33.738 & 94.482 & & 33.633 & 92.442 \\
\hline 800 & & 38.53 & 107.186 & & 37.219 & 99.135 & & 36.954 & 97.082 \\
\hline 900 & & 40.338 & 111.826 & & 37.971 & 103.488 & & 37.923 & 101.427 \\
\hline 1000 & & 41.907 & 116.155 & & 40.787 & 107.573 & & 40.63 & 105.509 \\
\hline 1100 & & 43.28 & 120.212 & & 41.12 & 111.419 & & 41.111 & 109.353 \\
\hline 1200 & & 44.486 & 124.027 & & 42.395 & 115.05 & & 42.399 & 112.984 \\
\hline 1300 & & 45.549 & 127.628 & & 43.509 & 118.485 & & 43.524 & 116.42 \\
\hline 1400 & & 46.487 & 131.036 & & 44.484 & 121.744 & & 44.507 & 119.68 \\
\hline 1500 & & 47.315 & 134.27 & & 46.4 & 124.84 & & 46.368 & 122.778 \\
\hline 2000 & & 50.25 & 148.317 & & 48.328 & 138.329 & & 48.364 & 136.276 \\
\hline 2500 & & 51.93 & 159.722 & & 50.009 & 149.306 & & 50.041 & 147.261 \\
\hline 3000 & & 52.953 & 169.285 & & 51.023 & 158.517 & & 51.05 & 156.478 \\
\hline 3500 & & 53.614 & 177.5 & & 51.674 & 166.433 & & 51.696 & 164.398 \\
\hline 4000 & & 54.062 & 184.689 & & 52.114 & 173.363 & & 52.132 & 171.33 \\
\hline 4500 & & 54.379 & 191.075 & & 52.424 & 179.519 & & 52.439 & 177.488 \\
\hline 5000 & & 54.61 & 196.816 & & 52.65 & 185.054 & & 52.662 & 183.025 \\
\hline
\end{tabular}

a Thermodynamic properties refer to the standard state of an ideal gas at 1 atm. $\mathrm{S}^{\circ}(\mathrm{T})$ and $\mathrm{C}^{\circ} \mathrm{p}(\mathrm{T})$ in $\mathrm{cal} \mathrm{mol}^{-1} \mathrm{~K}^{-1}$. 
Table 6. Ideal gas-phase thermodynamic property vs. temperature ${ }^{\mathrm{a}}$ of radicals of parent $\mathrm{CH} 3 \mathrm{CONH} 2$.

\begin{tabular}{|c|c|c|c|c|c|c|}
\hline \multirow{2}{*}{$\mathrm{T}(\mathrm{K})$} & \multirow{2}{*}{$\begin{array}{c}\Delta \mathrm{H}_{\mathrm{f}}{ }^{\circ} 298 \mathrm{kcal} \\
\mathrm{mol}^{-1}\end{array}$} & \multicolumn{2}{|c|}{$\mathrm{C} \bullet \mathrm{H}_{2}-\mathrm{C}=\mathrm{ONH}_{2}$} & \multirow{2}{*}{$\begin{array}{c}\Delta \mathrm{H}_{\mathrm{f}}{ }^{\circ} 298 \mathrm{kcal} \\
\mathrm{mol}^{-1}\end{array}$} & \multicolumn{2}{|c|}{$\mathrm{CH}_{3}-\mathrm{C}=\mathrm{ON} \bullet \mathrm{H}$} \\
\hline & & $\mathrm{Cp}(\mathrm{T})$ & $S^{\circ}(T)$ & & $\mathrm{Cp}(\mathrm{T})$ & $S^{\circ}(T)$ \\
\hline 1 & \multirow{26}{*}{-10.23} & 7.949 & 17.966 & \multirow{26}{*}{1.68} & 7.949 & 15.81 \\
\hline 51 & & 8.029 & 49.073 & & 7.952 & 46.907 \\
\hline 101 & & 9.422 & 54.969 & & 8.386 & 52.495 \\
\hline 151 & & 11.766 & 59.214 & & 9.781 & 56.132 \\
\hline 201 & & 14.198 & 62.928 & & 11.554 & 59.18 \\
\hline 251 & & 16.459 & 66.337 & & 13.404 & 61.952 \\
\hline 298 & & 18.432 & 69.393 & & 16.18 & 64.453 \\
\hline 400 & & 22.024 & 75.332 & & 19.761 & 69.423 \\
\hline 500 & & 24.848 & 80.554 & & 22.802 & 73.938 \\
\hline 600 & & 27.121 & 85.286 & & 25.331 & 78.138 \\
\hline 700 & & 28.985 & 89.607 & & 26.439 & 82.046 \\
\hline 800 & & 30.555 & 93.578 & & 29.212 & 85.691 \\
\hline 900 & & 31.907 & 97.254 & & 29.733 & 89.1 \\
\hline 1000 & & 33.088 & 100.675 & & 32.039 & 92.299 \\
\hline 1100 & & 34.126 & 103.875 & & 32.179 & 95.309 \\
\hline 1200 & & 35.043 & 106.882 & & 33.167 & 98.15 \\
\hline 1300 & & 35.854 & 109.718 & & 34.028 & 100.837 \\
\hline 1400 & & 36.573 & 112.4 & & 34.78 & 103.385 \\
\hline 1500 & & 37.21 & 114.944 & & 36.434 & 105.806 \\
\hline 2000 & & 39.482 & 125.985 & & 37.724 & 116.342 \\
\hline 2500 & & 40.794 & 134.945 & & 38.998 & 124.906 \\
\hline 3000 & & 41.597 & 142.457 & & 39.763 & 132.087 \\
\hline 3500 & & 42.117 & 148.91 & & 40.252 & 138.255 \\
\hline 4000 & & 42.47 & 154.557 & & 40.582 & 143.652 \\
\hline 4500 & & 42.72 & 159.574 & & 40.814 & 148.445 \\
\hline 5000 & & 42.902 & 164.085 & & 40.983 & 152.754 \\
\hline
\end{tabular}

a Thermodynamic properties refer to the standard state of an ideal gas at 1 atm. $\mathrm{S}^{\circ}(\mathrm{T})$ and $\mathrm{C}^{\circ} \mathrm{p}(\mathrm{T})$ in $\mathrm{cal} \mathrm{mol}^{-1} \mathrm{~K}^{-1}$.

Table 7. Ideal gas-phase thermodynamic property vs. temperature ${ }^{\mathrm{a}}$ of radicals of parent $\mathrm{CH} 3 \mathrm{CONHCH} 3$.

\begin{tabular}{|c|c|c|c|c|c|c|c|c|c|}
\hline \multirow{2}{*}{$\mathbf{T}(\mathbf{K})$} & \multirow{2}{*}{$\begin{array}{c}\Delta \mathrm{H}_{\mathrm{f}}{ }^{\circ} 298 \\
\mathrm{kcal} \mathrm{mol}^{-1}\end{array}$} & \multicolumn{2}{|c|}{$\mathrm{C} \cdot \mathrm{H}_{2}-\mathrm{C}=\mathrm{ONHCH}_{3}$} & \multirow{2}{*}{$\begin{array}{c}\Delta \mathrm{H}_{\mathrm{f}}{ }^{\circ} 298 \\
\mathrm{kcal} \mathrm{mol}^{-1}\end{array}$} & \multicolumn{2}{|c|}{$\mathrm{CH}_{3}-\mathrm{C}=\mathrm{ON} \cdot \mathrm{CH}_{3}$} & \multirow{2}{*}{$\begin{array}{c}\Delta \mathrm{H}_{\mathrm{f}}{ }^{\circ} 298 \\
\mathrm{kcal} \mathrm{mol}^{-1}\end{array}$} & \multicolumn{2}{|c|}{$\mathrm{CH}_{3}-\mathrm{C}=\mathrm{ONHC} \bullet \mathrm{H}_{2}$} \\
\hline & & $\mathrm{Cp}(\mathrm{T})$ & $\mathrm{S}^{\circ}(\mathrm{T})$ & & $\mathrm{Cp}(\mathrm{T})$ & $S^{\circ}(T)$ & & $\mathrm{Cp}(\mathrm{T})$ & $S^{\circ}(T)$ \\
\hline 1 & \multirow{26}{*}{-8.85} & 7.949 & 18.06 & \multirow{26}{*}{-4.02} & 7.949 & 15.856 & \multirow{26}{*}{-15.11} & 7.949 & 18.072 \\
\hline 51 & & 8.762 & 49.415 & & 8.619 & 47.154 & & 9.372 & 49.785 \\
\hline 101 & & 10.951 & 56.118 & & 10.268 & 53.62 & & 11.973 & 57.054 \\
\hline 151 & & 13.531 & 61.034 & & 12.061 & 58.114 & & 14.58 & 62.399 \\
\hline 201 & & 16.137 & 65.279 & & 13.97 & 61.837 & & 17.073 & 66.931 \\
\hline 251 & & 18.685 & 69.147 & & 16.06 & 65.17 & & 19.506 & 70.996 \\
\hline 298 & & 22.115 & 72.628 & & 21.367 & 68.168 & & 22.907 & 74.613 \\
\hline 400 & & 26.987 & 79.518 & & 25.829 & 74.199 & & 27.577 & 81.691 \\
\hline 500 & & 31.217 & 85.77 & & 29.988 & 79.812 & & 31.654 & 88.051 \\
\hline 600 & & 34.808 & 91.596 & & 33.633 & 85.148 & & 35.129 & 93.943 \\
\hline 700 & & 36.841 & 97.034 & & 34.446 & 90.202 & & 37.056 & 99.42 \\
\hline 800 & & 40.427 & 102.12 & & 39.434 & 94.979 & & 40.587 & 104.531 \\
\hline 900 & & 41.654 & 106.89 & & 39.552 & 99.493 & & 41.755 & 109.315 \\
\hline 1000 & & 44.584 & 111.376 & & 43.74 & 103.763 & & 44.654 & 113.81 \\
\hline 1100 & & 45.266 & 115.606 & & 43.342 & 107.806 & & 45.306 & 118.046 \\
\hline 1200 & & 46.732 & 119.605 & & 44.863 & 111.64 & & 46.754 & 122.048 \\
\hline 1300 & & 48.014 & 123.394 & & 46.182 & 115.281 & & 48.023 & 125.838 \\
\hline 1400 & & 49.136 & 126.991 & & 47.329 & 118.743 & & 49.136 & 129.435 \\
\hline 1500 & & 51.118 & 130.413 & & 50.395 & 122.041 & & 51.114 & 132.857 \\
\hline 2000 & & 53.55 & 145.343 & & 51.759 & 136.457 & & 53.532 & 147.783 \\
\hline 2500 & & 55.472 & 157.513 & & 53.646 & 148.224 & & 55.455 & 159.949 \\
\hline 3000 & & 56.629 & 167.734 & & 54.77 & 158.109 & & 56.615 & 170.167 \\
\hline 3500 & & 57.37 & 176.521 & & 55.485 & 166.608 & & 57.358 & 178.952 \\
\hline 4000 & & 57.87 & 184.215 & & 55.965 & 174.049 & & 57.86 & 186.645 \\
\hline 4500 & & 58.221 & 191.052 & & 56.302 & 180.66 & & 58.214 & 193.48 \\
\hline 5000 & & 58.478 & 197.199 & & 56.547 & 186.605 & & 58.471 & 199.627 \\
\hline
\end{tabular}

a Thermodynamic properties refer to the standard state of an ideal gas at 1 atm. $\mathrm{S}^{\circ}(\mathrm{T})$ and $\mathrm{C}^{\circ} \mathrm{p}(\mathrm{T})$ in $\mathrm{cal} \mathrm{mol}^{-1} \mathrm{~K}^{-1}$. 
Entropy and heat capacity contributions of the parent molecules and radicals using VIBIR have been calculated at temperatures ranging from 1-5000 K.

\section{Summary}

Thermochemical properties are presented for acetic acid hydrazide, Acetamide, $\mathrm{N}$ methyl acetamide, and radicals that result from the loss of $\mathrm{H}$ atoms from the carbon and the nitrogen atoms. Standard enthalpies from all the work reactions and each of the calculation methods are in reasonably good agreement, suggesting that the B3LYP DFT calculations, in conjunction with the work reactions used here, are acceptable methods for larger hydrazide and amides. $\mathrm{C}-\mathrm{H}$ bond energy values for the radicals $\mathrm{C} \bullet \mathrm{H}_{2}-\mathrm{C}=\mathrm{ONHNH}_{2}, \mathrm{C} \cdot \mathrm{H}_{2}-\mathrm{C}=\mathrm{ONH}_{2}$ and $\mathrm{C}_{2} \mathrm{H}_{2}-\mathrm{C}=\mathrm{ONHCH}_{3}$ from the B3LYP / 6-31G $(\mathrm{d}, \mathrm{p}), \mathrm{B} 3 \mathrm{LYP} / 6-31 \mathrm{G}(2 \mathrm{~d}, 2 \mathrm{p})$ and CBS-QB3 levels of calculation were 99.50, 99.40, 99.61 and $100.22,99.43,99.52$ and 99.54, 99.42, 99.56 $\mathrm{kcal} \mathrm{mol}^{-1}$ respectively. The $\mathrm{N}-\mathrm{H}$ bond in the acetohydrazide was weak, at $78.17 \mathrm{kcal}$ $\mathrm{mol}^{-1}$, but strong in $\mathrm{N}$-methyl acetamide at $104.20 \mathrm{kcal} \mathrm{mol}^{-1}$. The $\mathrm{HN}-\mathrm{H}$ bond energies for the formation of the radicals $\mathrm{CH}_{3}-\mathrm{C}=\mathrm{ONHN} \bullet \mathrm{H}$ and $\mathrm{CH}_{3}-\mathrm{C}=\mathrm{ON} \bullet \mathrm{H}$ from the parent molecules were also similar across the different B3LYP basis sets and CBS-QB3 level of calculations $\left(\mathrm{CH}_{3}-\mathrm{C}=\mathrm{ONHN} \bullet \mathrm{H}=81.93,81.45,82.13 ; \mathrm{CH}_{3}-\mathrm{C}=\mathrm{ON} \bullet \mathrm{H}=111.74,110.76\right.$, $111.99 \mathrm{kcal} \mathrm{mol}^{-1}$ ) respectively.

Supplementary Materials: The following are available online at https:/ /www.mdpi.com/2673-7 264/1/1/2/s1. Table S1. $\mathrm{CH}_{3} \mathrm{CONHNH}_{2}$. Table S2. $\mathrm{CH}_{3} \mathrm{CONH}_{2}$. Table S3. $\mathrm{CH}_{3} \mathrm{CONHCH}_{3}$. Table S4. Moments of Inertia (amu bohr [2]). Table S5. Vibration Frequencies $\left(\mathrm{cm}^{-1}\right)$. Table S6. Mulliken Atomic Charges for Acetohydrazide and its Radicals. Table S7. Mulliken Atomic Charges for Acetamide and its Radicals. Table S8. Mulliken Atomic Charges for N-Methyl Acetamide and its Radicals.

Author Contributions: Conceptualization, S.C. and J.W.B.; Formal analysis, S.C., and J.W.B.; Methodology, S.C., and J.W.B.; Software, J.W.B.; Writing-original draft, S.C.; Resources, S.C. and J.W.B.; Data Curations, S.C.; Writing-Review and Editing, S.C. and J.W.B.; Supervision, J.W.B. All authors have read and agreed to the published version of the manuscript.

Funding: This research received no external funding.

Institutional Review Board Statement: Not applicable.

Informed Consent Statement: Not applicable.

Conflicts of Interest: The authors declare no conflict of interest.

\section{References}

1. Mennucci, B.; Martínez, J.M. How to model solvation of peptides? Insights from a quantum-mechanical and molecular dynamics study of N-methylacetamide. 1. Geometries, infrared, and ultraviolet spectra in water. J. Phys. Chem. B 2005, 109, 9818-9829. [CrossRef] [PubMed]

2. Ataka, S.; Takeuchi, H.; Tasumi, M. Infrared studies of the less stable cis form of N-methylformmaide and N-methylacetamide in low temperature nitrogen matrices and vibrationa analyses of the trans and cis forms of these molecules. J. Mol. Struct. 1984, 113, 147-160. [CrossRef]

3. Mayne, L.C.; Hudson, B. Resonance Rama Spectroscopy of N-methylacetamide: Overtones and combinations of the carbonnitrogen stretch (aminde II) and effect of solvation on the carbon. J. Phys. Chem. 1991, 95, 2962. [CrossRef]

4. Chen, X.G.; Schweitzerstenner, R.; Asher, S.A.; Mirkin, N.G.; Krimms, S. Vibrationals assignments of trans-N-methylacetamide and some of its deuterated isotopomers from band decomposition of IR, visible, and resonance Raman spectra. J. Phys. Chem. 1995, 99, 3074. [CrossRef]

5. Torii, H.; Tasumi, T.; Tasumi, M. Effects of hydration on the structure, vibrational wavenumbers, vibrational force field and resonance raman intensities of N-methylacetamide. J. Raman Spectrosc. 1998, 29, 537. [CrossRef]

6. Kubelka, J.; Keiderling, T.A. Ab Initio Calculation of Amide Carbonyl Stretch Vibrational Frequencies in Solution with Modified Basis sets.1. N-methylacetamide. J. Phys. Chem. A 2001, 105, 10922. [CrossRef]

7. Cuevas, G.; Renugopalakrishnan, V.; Madrid, G.; Hagler, A.T. Density function studies of peptides Part I. Vibrational frequencies including isotopic effects and NMR chemical shifts of N-methylacetamide, a pedtide model from density fucntion and MP2 calculations. Phys. Chem. Chem. Phys. 2002, 4, 1490. [CrossRef]

8. Papamokos, G.V.; Demetropoulos, I.N. Vibrational Frequencies of Amides and Amide Dimers: The Assessment of PW91XC Functional. J. Phys. Chem. A 2004, 108, 7291. [CrossRef] 
9. Zhang, R.; Li, H.R.; Lei, Y.; Han, S.J. Structures and interactions in N-methylacetamide-water mixtures studied by IR spectra and density functional theory. J. Mol. Struct. 2004, 693, 17. [CrossRef]

10. Kaledin, A.L.; Bowman, J.M. Full Dimensional Quantum Calculations of Vibrationals Energies of N-methylacetamide. J. Phys. Chem. A 2007, 111, 5593-5598. [CrossRef] [PubMed]

11. Badawi, H.M. Vibrational Spectra and analysis of acetohydrazide CH3-CO-NH-NH2. Spectrochim. Acta Part A Mol. Biomol. Spectrosc. 2007, 67, 592-597. [CrossRef]

12. Jasien, P.G.; Stevens, W.J.; Krauss, M. Ab Initio calculations of the rotational barriers in formamide and acetamide: The effects of polarization functions and correlation. J. Mol. Struct. THEOCHEM 1986, 139, 197-206. [CrossRef]

13. Zhou, Y.; Yang, Z.; Zhang, C.; Liu, X. Reaction mechanisms of DNT with hydroxyl radicals for advanced oxidation processes DFT study. J. Mol. Modeling 2017, 23, 139. [CrossRef]

14. Frisch, M.J.; Trucks, G.W.; Schlegel, H.B.; Scuseria, G.E.; Robb, M.A.; Cheeseman, J.R.; Zakrzewski, V.G.; Stratmann, J.A.; Montgomery, R.E., Jr.; Burant, J.C.; et al. Gaussian 98; Gaussian, Inc.: Pittsburgh, PA, USA, 1998.

15. Frisch, M.J.; Trucks, G.W.; Schlegel, H.B.; Scuseria, G.E.; Robb, M.A.; Cheeseman, J.R.; Scalmani, G.; Barone, V.; Mennucci, B.; Petersson, G.A.; et al. Gaussian 09; Gaussian, Inc.: Wallingford, CT, USA, 2009.

16. Becke, A.D. Density-functional Thermochemistry. III. The Role of Exact Exchange. J. Chem. Phys. 1993, 98, 5648-5652. [CrossRef]

17. Lee, C.; Yang, W.; Parr, R.G. Development of the Colle-Salvetti Correlation-Energy Formula Into a Functional of the Electron Density. Phys. Rev. B Condens. Matter Mater. Phys. 1988, 37, 785-789. [CrossRef]

18. Purnell, D.L., Jr.; Bozzelli, J.W. Thermochemical Properties: Enthalpy, Entropy, and Heat Capacity of C2-C3 Fluorinated Aldehydes. Radicals and Flurocarbon Group Additivity. J. Phys. Chem. A 2019, 123, 650-665. [CrossRef]

19. Montgomery, J.A.; Frisch, M.J.; Ochterski, J.W.; Petersson, G.A. A Complete Basis Set Model Chemistry. VII. Use of the Minimum Population Localization Method. J. Chem. Phys. 2000, 112, 6532-6542. [CrossRef]

20. Zhu, L.; Bozzelli, J.W. The multi-channel reaction of CH3S+ 3O2: Thermochemistry and Kinetik barriers. J. Mol. Struct. THEOCHEM 2005, 728, 147-157. [CrossRef]

21. NIST Chemistry WebBook. Standard Reference Database No. 69. In February 2000.. Available online: http:/ / webbook.nist.gov / chemistry/.NIST (accessed on 15 April 2011).

22. Dorofeeva, O.V.; Ryzhova, O.N.; Suchkova, T.A. Enthalpies of Formation of Hydrazine and Its Derivatives. J. Phys. Chem. A 2017, 121, 5361. [CrossRef] [PubMed]

23. Pedley, J.B. Thermochemical Data and Structures of Organic Compounds; Thermodynamics Research Center: College Station, TX, USA, 1994; Volume I.

24. Anderson, W.R. Oscillator strengths of amidogen and the heats of formation of imidogen and amidogen. J. Phys. Chem. 1989, 93, 530-536. [CrossRef]

25. Wang, H.; Castillo, A.; Bozzelli, J.W. Thermochemical Properties Enthalpy, Entropy, and Heat Capacity of C1-C4 Fluorinated Hydrocarbons: Flurocarbon Group Additivity. J. Phys. Chem. A 2015, 119, 8202-8215. [CrossRef]

26. Ruscic, B. ATcT Enthalpies of Formation Based on Version 1.112 of the Thermochemical Network. Available online: http: / /atct.anl.gov/Thermochemical_Data/version_1.112/ (accessed on 15 April 2011).

27. Zhao, Y.; Frederick; Bordwell, G.; Cheng, J.-P.; Wang, D. Equilibrium Acidities and Homolytic Bond Dissociation Energies (BDEs) of the Acidic H-N Bonds in the Hydrazines and Hydrazides. J. Am. Chem. Soc. 1997, 119, 9125-9129. [CrossRef]

28. Richard, J.; Williams, G.; O’Donoghu, A.; Amyes, T.L. Formation and Stability of Enolates of Acetamide and Acetate Anion: An Eigen Plot for Proton Transfer at alpha-Carbonyl Carbon. J. Am. Chem. Soc. 2002, 124, 2957-2968. [CrossRef]

29. Ledneczki, I.; Forgo, P.; Kiss, J.T.; Molnar, A.; Palinko, I. Conformational behavious of acetamide derivatives studies by NMR spectroscopic and computational methods. J. Mol. Struct. 2007, 834-836, 349-354. [CrossRef]

30. Bailey, J.R.; McMahon, T.J.; Bird, R.G. Dyanamics of peptide bonds: A study of N-methylethanolamine using chirped-pulsed Fourier transform microwave spectroscopy. J. Mol. Spectrosc. 2017, 335, 33-36. [CrossRef]

31. Asatryan, R.; Bozzelli, J.W.; Simmie, J.M. Thermochemistry for enthalpies and reaction paths for nitrous acid isomers. Int. J. Chem. Kinet. 2007, 39, 378-398. [CrossRef]

32. Sun, H.; Bozzelli, J.W. Structures, Intramolecular Rotation Barriers, and Thermochemical Properties of Radicals Derived from H Atom Loss in Mono-, Di-, and Trichloromethanol and Parent Chloromethanols. J. Phys. Chem. A 2001, 105, 4504-4516. [CrossRef]

33. Pitzer, K.S. Thermochemistry and bond energies of nitro-alkanes, -alkenes, -carbonyls and corresponding nitrites. J. Chem. Phys. 1937, 5, 469. [CrossRef]

34. Pitzer, K.S.; Gwinn, W.D. Energy levels and thermodynamic functions for molecules with internal rotation I. Rigid frame with attached tops. J. Chem. Phys. 1942, 10, 428. [CrossRef]

35. Sheng, C. Elementary, Pressure Dependent Model for Combustion of C1, C2 and Nitrogen Contaninig Hydrocarbons: Operation of a Pilot Scale Incinerator and Model Comparison. Ph.D. Thesis, Chemical Engineering, New Jersey Institute of Technology, Newark, NJ, USA, 2002. 